\title{
Society for Immunotherapy of Cancer (SITC) clinical practice guideline on immunotherapy for the treatment of hepatocellular carcinoma
}

\author{
Tim F Greten (D) , ${ }^{1}$ Ghassan K Abou-Alfa, ${ }^{2,3}$ Ann-Lii Cheng, ${ }^{4}$ Austin G Duffy, ${ }^{5}$ \\ Anthony B. El-Khoueiry, ${ }^{6}$ Richard S Finn, ${ }^{7}$ Peter R Galle, ${ }^{8}$ Lipika Goyal, ${ }^{9}$ \\ Aiwu Ruth He, ${ }^{10}$ Ahmed O Kaseb, ${ }^{11}$ Robin Kate Kelley, ${ }^{12}$ Riccardo Lencioni, ${ }^{13,14}$ \\ Amaia Lujambio, ${ }^{15}$ Donna Mabry Hrones, ${ }^{1}$ David J Pinato (D) , ${ }^{16}$ Bruno Sangro, ${ }^{17,18}$ \\ Roberto I Troisi, ${ }^{19}$ Andrea Wilson Woods, ${ }^{20}$ Thomas Yau, ${ }^{21}$ Andrew X Zhu, ${ }^{9,22}$ \\ Ignacio Melero (1) 17,23,24
}

To cite: Greten TF, AbouAlfa GK, Cheng A-L, et al. Society for Immunotherapy of Cancer (SITC) clinical practice guideline on immunotherapy for the treatment of hepatocellular carcinoma. Journal for ImmunoTherapy of Cancer 2021;9:e002794. doi:10.1136/ jitc-2021-002794

Accepted 02 July 2021
Check for updates

(c) Author(s) (or their employer(s)) 2021. Re-use permitted under CC BY-NC. No commercial re-use. See rights and permissions. Published by BMJ.

For numbered affiliations see end of article.

\section{Correspondence to Dr Ignacio Melero; imelero@unav.es}

Dr Tim F Greten; tim.greten@nih.gov

\section{ABSTRACT}

Patients with advanced hepatocellular carcinoma (HCC) have historically had few options and faced extremely poor prognoses if their disease progressed after standardof-care tyrosine kinase inhibitors (TKIs). Recently, the standard of care for HCC has been transformed as a combination of the immune checkpoint inhibitor (ICl) atezolizumab plus the anti-vascular endothelial growth factor (VEGF) antibody bevacizumab was shown to offer improved overall survival in the first-line setting. Immunotherapy has demonstrated safety and efficacy in later lines of therapy as well, and ongoing trials are investigating novel combinations of ICls and TKls, in addition to interventions earlier in the course of disease or in combination with liver-directed therapies. Because HCC usually develops against a background of cirrhosis, immunotherapy for liver tumors is complex and oncologists need to account for both immunological and hepatological considerations when developing a treatment plan for their patients. To provide guidance to the oncology community on important concerns for the immunotherapeutic care of HCC, the Society for Immunotherapy of Cancer (SITC) convened a multidisciplinary panel of experts to develop a clinical practice guideline (CPG). The expert panel drew on the published literature as well as their clinical experience to develop recommendations for healthcare professionals on these important aspects of immunotherapeutic treatment for HCC, including diagnosis and staging, treatment planning, immune-related adverse events (irAEs), and patient quality of life (QOL) considerations. The evidence- and consensus-based recommendations in this CPG are intended to give guidance to cancer care providers treating patients with HCC.

\section{INTRODUCTION}

Hepatocellular carcinoma (HCC) is the most common primary cancer of the liver and is among the top causes of cancer-related death worldwide. ${ }^{1}$ Mortality due to HCC exerts a high human toll in most countries around the world, and in the United States (US), the incidence has increased markedly in recent years. ${ }^{2}$ Risk factors for HCC are unevenly distributed around the globe. In the USA, Europe, and Japan, the predominant risk factors for HCC are overweight-related and obesity-related conditions, for example, non-alcoholic fatty liver disease (NAFLD), as well as hepatitis C virus $(\mathrm{HCV})$, and alcohol abuse, ${ }^{3}$ whereas in eastern Asia and sub-Saharan Africa hepatitis $\mathrm{B}$ virus $(\mathrm{HBV})$ is more prevalent as an etiological agent. Additional risk factors include diabetes mellitus, obesity, exposure to aflatoxin $\mathrm{B}$, hemachromatosis, and other hereditary disorders. ${ }^{45}$

Although curative interventions such as liver transplant, surgery, and ablation may offer favorable outcomes for patients with early-stage HCC, for many years options were limited and prognosis was very poor for advanced disease. ${ }^{6-8}$ The 2007 approval of the multi-tyrosine kinase inhibitor (TKI), sorafenib, for the first-line treatment of advanced HCC represented a breakthrough as it was the first systemic therapy in several decades to demonstrate improved survival in liver cancer. $^{9}$ However, despite several additional approvals for TKIs including regorafenib $^{10}$ and lenvantinib ${ }^{11}$ in the subsequent years, ${ }^{12}$ the new modalities only offered incremental increases in overall survival (OS) for patients with advanced HCC, until the advent of immunotherapy and immune-based combination therapies.

In 2017, the US Food and Drug Administration (FDA) granted the first approval for an immune checkpoint inhibitor (ICI) for HCC. Nivolumab (targeting programmed 
cell death protein 1 [PD-1]) monotherapy received accelerated approval based on a significant response rate and prolonged duration of response (DOR) with manageable side effects in patients who had previously been treated with sorafenib. ${ }^{13}$ This was followed by encouraging data for other ICIs-pembrolizumab (another anti-PD-1 ICI) monotherapy ${ }^{14}$ and nivolumab in combination with the cytotoxic T lymphocyte antigen-4 (CTLA-4) antagonist ipilimumab ${ }^{15}$-resulting in further accelerated approvals by the FDA. The confirmatory phase III studies for singleagent nivolumab and permbrolizumab, however, did not meet their end points. In 2020, the anti-programmed death-ligand 1 (PD-L1) antibody atezolizumab in combination with the anti-vascular endothelial growth factor (VEGF) antibody bevacizumab ${ }^{16}$ gained full FDA approval for first-line treatment of HCC on the basis of the phase III study IMbrave150. This was the first regimen to demonstrate superiority to sorafenib in HCC since sorafenib's approval in 2007, in addition to being the first immunotherapy plus anti-VEGF combination to gain approval for liver cancer. Additional trials are ongoing and the therapeutic landscape continues to evolve and expand.

HCC often develops on a background of chronic inflammation, metabolic stress, cirrhosis, or fibrosis, and thus, the use of immunotherapy in the setting of a compromised liver is a complex but common challenge. Although HCC is frequently an immunogenic cancer, characterized by tumor-infiltrating lymphocytes (TILs) in the tumor microenvironment, the intratumoral milieu has been shown to be generally immunosuppressive-in part due to the acquired immune dysfunction that occurs with cirrhosis, viral infection, or environmental insults that contribute to disease development, but also partially related to the liver's intrinsic tolerogenicity. ${ }^{17} 18$ Despite these hurdles, the incorporation of immunotherapy into HCC care has offered more options to clinicians and has extended survival considerably for a subset of patients.

The approval of immunotherapy agents for the treatment of HCC is relatively recent as compared with other malignancies and experience with these new therapies is still limited. Additionally, immunotherapy carries unique considerations in many clinical aspects including patient selection, management of immune-related adverse events (irAEs), and evaluation of response to therapy compared with other systemic treatments. To support the oncology community and provide evidence- and consensus-based recommendations on immunotherapy for HCC, the Society for Immunotherapy of Cancer (SITC) convened an international panel of experts to develop a new clinical practice guideline $(\mathrm{CPG})$, covering topics including recommended therapies, emerging agents, diagnostics and biomarkers, monitoring response to treatment, special patient populations, toxicity management, and quality of life (QOL). Although the guideline focuses on therapies approved by the FDA, the authors, as an international team, acknowledge that recommendations may not fully align with approval or reimbursement policies in other countries outside the US, and they encourage harmonization. The recommendations within this guideline are meant to complement rather than supplant sound clinical judgment, and their aim is to provide clinicians with the most current thinking on integrating immunotherapy into the treatment of patients with HCC.

\section{GUIDELINE DEVELOPMENT METHODS}

The Institute of Medicine's (IOM) Standards for Developing Trustworthy Clinical Practice Guidelines were used as a model to develop the recommendations in this manuscript. IOM standards dictate that guideline development is led by a multidisciplinary expert panel using a transparent process where both funding sources and conflicts of interest are readily reported. This CPG is intended to provide guidance and is not a substitute for the professional judgment of individual treating physicians.

\section{Conflict of interest management}

As outlined by IOM standards, all financial relationships of expert panel members that might result in actual, potential, or perceived conflicts of interest were individually reported. Disclosures were made prior to the onset of manuscript development and updated on an annual basis. In addition, panel members were asked to articulate any actual or potential conflicts at all key decision points during guideline development, so that participants would understand all possible influences, biases, and/ or the diversity of perspectives on the panel. Although some degree of relationships with outside interests are to be expected among experts, panel candidates with significant financial connections that may compromise their ability to fairly weigh evidence (either actual or perceived) were not eligible to participate in guideline development.

Recognizing that guideline panel members are among the leading experts on the subject matter under consideration and guideline recommendations should have the benefit of their expertize, any identified potential conflicts of interests were managed as outlined in SITC's disclosure and conflict of interest resolution policies. As noted in these policies, panel members disclosing a real or perceived potential conflict of interest may be permitted to participate in consideration and decisionmaking of a matter related to that conflict, but only if deemed appropriate after discussion and agreement by the expert panel.

The financial support for the development of this guideline was provided solely by SITC. No commercial funding was received.

\section{Recommendation development}

Panel recommendations are based on literature evidence, where possible, and clinical experience, where appropriate. ${ }^{19}$ Consensus for the recommendations here was generated by open communication and scientific debate in small-group and whole-group settings, surveying and 
Table 1 Summary of 'The Oxford Levels of Evidence 2' (Adapted from the OCEBM Levels of Evidence Working Group)

\begin{tabular}{lllll}
\hline Level 1 & Level 2 & Level 3 & Level 4 & Level 5 \\
\hline $\begin{array}{l}\text { Systematic review or } \\
\text { meta-analysis }\end{array}$ & $\begin{array}{l}\text { Randomized trial or } \\
\text { observational study } \\
\text { with dramatic effect }\end{array}$ & $\begin{array}{l}\text { Non-randomized, } \\
\text { controlled cohort, or } \\
\text { follow-up study }\end{array}$ & $\begin{array}{l}\text { Case series, case- } \\
\text { control, or historically reasoning } \\
\text { controlled study }\end{array}$ & Mechanism-based \\
\hline
\end{tabular}

OCEBM, Oxford Centre for Evidence-Based Medicine.

responses to clinical questionnaires, as well as formal voting in consensus meetings.

For transparency, a draft of this CPG was made publicly available for comment during the development process and prior to publication. All comments were evaluated and considered for inclusion into the final manuscript according to the IOM standard.

\section{Evidence rating}

The evidence- and consensus-based recommendations of the panel were refined throughout the development process in order to obtain the highest possible agreement among the experts, however, the minimum threshold was defined as $75 \%$ approval among the voting members. Evidence supporting panel recommendations was graded according to the Oxford Centre for Evidence-Based Medicine (OCEBM) Levels of Evidence Working Group 'The Oxford Levels of Evidence 2' (2016 version). A summary of the OCEBM grading scale may be found in table 1 . The level of evidence (LE) for a given recommendation is expressed in parentheses following the recommendation (eg, LE: 1). Recommendations without an associated LE were based on expert consensus.

\section{DIAGNOSTICS AND STAGING FOR PATIENTS WITH HCC Initial HCC diagnosis}

The initial diagnostic workup of HCC typically comprises a histologic analysis of tumor samples obtained by biopsy or surgery, cross-sectional imaging, a detailed analysis of the liver's condition with laboratory studies, and an assessment of the potential etiology of the HCC including investigations of $\mathrm{HBV}$ and $\mathrm{HCV}$ viral status. Guidelines for surveillance screening, initial diagnosis, and staging of HCC have been developed by multiple organizations including, but not limited to, the American Association for the Study of Liver Diseases (AASLD), ${ }^{7}$ the American College of Gastroenterology (AGC), ${ }^{20}$ the European Society for Medical Oncology (ESMO), ${ }^{21}$ the European Association for the Study of the Liver (EASL) ${ }^{22}$ and the Japan Society of Hepatology (JSH). ${ }^{23}$ These organizations and others have also put forth guidelines for nonimmunotherapeutic approaches for the treatment of HCG.

HCC may be identified using computerized tomography (CT) and magnetic resonance imaging (MRI) with Liver Imaging Reporting And Data Systems (LIRADS) ${ }^{24}$ The LI-RADS system provides a standardized approach for radiologists to communicate with the treating physicians and provides a certain level of confidence that a lesion in a cirrhotic liver or a liver at risk for cirrhosis presents as HCC on imaging. ${ }^{25}$ LI-RADS staging ranges from LR-1, for lesions that are definitely benign, to LR-5, which represents 100\% probability of being HCC. The LI-RADS system acknowledges that limitations exist, and has included an LR-NC (for non-categorizable) category where diagnostic possibilities cannot be meaningfully narrowed. LI-RADS is endorsed by the AASLD, ${ }^{7}$ as well as by the Organ Procurement and Transplantation Network (OPTN)/United Network for Organ Sharing (UNOS). Contrast agents have also greatly enhanced the diagnostic accuracy of MRIs. Multiple meta-analyses have determined that gadolinium ethoxybenzyl diethylenetriamine pentaacetic acid (Gd-EOB-DTPA)-enhanced MRI has superior sensitivity, specificity, and diagnostic odds ratio (OR) as compared with multidetector $\mathrm{CT}^{26-28}$ However, most published guidelines do not recommend one imaging modality over the other.

Patients with HCG often present with underlying cirrhosis-two conditions with independent mortality risks. It is essential for a care team comprised of multiple specialties, including perspectives from both hepatology and oncology, to be established early so that a treatment plan that addresses all of the complex needs of a patient with HCC may be developed. ${ }^{29}$ A multidisciplinary tumor board review of liver lesions is recommended for HCC diagnosis and management plans, particularly for patients with tumors that may be eligible for transplant, surgery, or liver-directed treatments.

Historically, avoiding tumor biopsy has been acceptable practice in patients with cirrhosis and imaging characteristics consistent with HCC. One concern of performing biopsies in this disease has been the putative risk for tumor dissemination outside the liver via needle track seeding. The occurrence of needle track seeding appears to be uncommon in the published literature, however, with incidence rates estimated to be as low as $2.7 \%$ overall, or $0.9 \%$ per year. ${ }^{30}$ While biopsy may be less encouraged in certain clinical scenarios such as in patients where liver transplants are being considered, histologic diagnosis is increasingly encouraged for the diagnosis of HCC, particularly for more advanced tumors requiring systemic therapy. Other primary liver tumors such as cholangiocarcinoma or mixed cholangiohepatoma can occasionally present very similarly to HCC, and the treatment for these tumors can be distinct. Other entities such as metastatic neuroendocrine cancers can similarly demonstrate 
Table 2 Radiographic T-staging by LI-RADS and OPTN/ UNOS $^{236}$

\begin{tabular}{ll}
\hline Stage & Definition \\
\hline 0 & No HCC \\
1 & One HCC $<20 \mathrm{~mm}$ \\
2 & $\begin{array}{l}\text { One HCC } \geq 20 \mathrm{~mm} \text { and } \leq 50 \mathrm{~mm} \text {, or two or three } \\
\text { HCCs, all } \leq 30 \mathrm{~mm}\end{array}$ \\
3 & $\begin{array}{l}\text { One HCC }>50 \mathrm{~mm} \text {, or two or three HCCs, at least } \\
\text { one }>30 \mathrm{~mm}\end{array}$ \\
4 & $\begin{array}{l}\text { 4A. Four or more HCCs, regardless of size } \\
\text { 4B. HCC }+ \text { TIV }\end{array}$
\end{tabular}

HCC, hepatocellular carcinoma; LI-RADS, Liver Imaging Reporting And Data System; OPTN/UNOS, Organ Procurement and Transplantation Network/United Network for Organ Sharing; TIV, tumor in vein.

arterial enhancement on multiphase imaging. ${ }^{31}$ Additionally, in rare instances, tissue biopsy may uncover certain genetic alterations that render a patient eligible for a tissue-agnostic therapy or a clinical trial.

\section{HCC staging}

An ideal staging system in HCC serves two purposes: treatment indication and prognostic prediction. A variety of staging systems have been developed, and their performance and validation varies. While some staging systems focus on pathology, others incorporate radiological characteristics, serum biomarkers, liver function, and performance status. In most solid tumors, staging is performed at the time of surgery using resected specimens. The Tumor-Node-Metastases (TNM) classification, developed by the American Joint Committee on Cancer (AJCC), classifies the primary tumor $(\mathrm{T})$ based on size, number, and vascular invasion. ${ }^{32}$ However, the TNM classification is not currently used to guide treatment for HCC. Also, importantly, the TNM classification should not be confused with the radiologic T-staging system used by LI-RADS and OPTN/UNOS, which is summarized in table 2.

Radiographic T-staging is of limited pretreatment prognostic and predictive value for patients being considered for systemic therapy, as the system does not take into account liver function, which is an important risk factor for patients with HCC. Several alternative staging or scoring systems have been developed, including the Barcelona-Clinic Liver Cancer (BCLC) system, ${ }^{33}$ Cancer of the Liver Italian Program (CLIP),${ }^{34}$ Japan Integrated Staging (JIS), ${ }^{35}$ Chinese University Prognostic Index (CUPI) ${ }^{36}$ Groupe d'Etude et de Traitement du Carcinome Hépatocellulaire $(\mathrm{GETCH})^{37}$ plus many others.

The BCLC system, summarized in table 3 , has gained wide recognition and has been endorsed by multiple international hepatology associations including $\mathrm{AASLD}^{6}$ and EASL. Several large-scale cohort studies have validated the BCLC system, including in Korean patients with treatment-naive HCC, ${ }^{38}$ US patients, ${ }^{39}$ and Italian patients undergoing radical surgery. ${ }^{40}$ In addition, scoring
Table 3 Barcelona-Clínic Liver Cancer (BCLC) classification with stage definitions and typical survival outcomes

\begin{tabular}{|c|c|c|}
\hline & $\begin{array}{l}\text { Stage definition } \\
\text { (BCLC } 2018 \text { update) }\end{array}$ & $\begin{array}{l}\text { Estimated } \\
\text { survival }\end{array}$ \\
\hline $\begin{array}{l}\text { Stage } 0 \text {-Very } \\
\text { early-stage }\end{array}$ & $\begin{array}{l}\text { Single nodule } \leq 2 \mathrm{~cm} \text {; } \\
\text { ECOG PS } 0^{*} ; \\
\text { Preserved liver } \\
\text { function }\end{array}$ & $>5$ years \\
\hline $\begin{array}{l}\text { Stage A-Early- } \\
\text { stage }\end{array}$ & $\begin{array}{l}\text { Single or up to three } \\
\text { nodules } \leq 3 \mathrm{~cm} ; \\
\text { ECOG PS } 0^{*} ; \\
\text { Preserved liver } \\
\text { function }\end{array}$ & $>5$ years \\
\hline $\begin{array}{l}\text { Stage B- } \\
\text { Intermediate-stage }\end{array}$ & $\begin{array}{l}\text { Multinodular; } \\
\text { ECOG PS 0*; } \\
\text { Preserved liver } \\
\text { function }\end{array}$ & $>2$ to 5 years \\
\hline $\begin{array}{l}\text { Stage C- } \\
\text { Advanced-stage }\end{array}$ & $\begin{array}{l}\text { Portal invasion; } \\
\text { Extrahepatic spread; } \\
\text { ECOG PS 1-2; } \\
\text { Preserved liver } \\
\text { function }\end{array}$ & $>1$ year \\
\hline $\begin{array}{l}\text { Stage D-Terminal- } \\
\text { stage }\end{array}$ & $\begin{array}{l}\text { ECOG PS 3-4; } \\
\text { End-stage liver } \\
\text { function }\end{array}$ & 3 months \\
\hline
\end{tabular}

*The American Association for the Study of Liver Disease (AASLD) recommends including ECOG PS 0 to 1 in stage 0 , A and B, because of the significant overlap between PS 0 and PS 1 BCLC, Barcelona-Clinic Liver Cancer; ECOG, Eastern Cooperative Oncology Group; PS, performance status.

by the BCLC system has been reported and studied in subgroup analyses for most of the phase III studies done in advanced HCC.

HCC is a highly heterogeneous disease with varied underlying etiologies depending on geography and demographics. Studies comparing the performance of staging systems for predicting prognosis have returned conflicting results depending on the patient population investigated and the treatments administered. The JIS score showed the best ability to predict OS by disease stage in an analysis of Japanese patients, ${ }^{41}$ whereas an analysis of 1,713 prospectively enrolled patients with HCC in Taiwan found that CLIP was the best prognostic model in patients undergoing both curative and non-curative treatments. ${ }^{42}$ In the advanced and metastatic disease setting, another comparison of the prognostic value of different systems determined CLIP and CUPI to be the most reliable staging systems for patients with HCV and HBV etiologies, respectively. ${ }^{43}$ In these analyses of patients with advanced disease in need for systemic therapy, BCLC and TNM lacked prognostic value.

Liver function assessment is a critical component of HCC treatment that is required for every patient. Some of the staging systems embed within them the Child-Pugh classification, recognizing the need for assessing the 
extent of liver functionality as part of the staging of the disease. The Child-Pugh score evolved over time from the original system built in 1973 to help assess for survival of patients with bleeding esophageal varices. ${ }^{44}$ Of note, in the original Pugh effort, none of the patients had HCC. The system evolved into a five-parameter staging system which consists of three laboratory values (serum albumin, bilirubin, and prothrombin levels) and two clinically assessed variables (presence and degree of ascites and hepatic encephalopathy). A final score ranging from 5 to 15 is calculated based on the range of laboratory values and severity of clinical symptoms, and then classified into one of three classes: A (5-6), B (7-9), and C (10-15). ${ }^{445}$ Median survival of untreated HCC has been shown to be approximately 2.5 times lower in patients with ChildPugh B disease compared with those with Child-Pugh A. ${ }^{46}$ Recently, however, the limitations and subjectivity involved in the grading of clinical variables have called into question Child-Pugh scores in assessing liver function in HCC. ${ }^{47}$

The albumin-bilirubin (ALBI) grade, a simpler model to assess liver function based only on serum albumin and bilirubin, has been validated in study cohorts from multiple geographic regions and multiple clinical scenarios, including patients undergoing resection and sorafenib treatment. The score is calculated as $\left(\log _{10}\right.$ bilirubin $\left.[\mu \mathrm{mol} / \mathrm{L}] \times 0.66\right)+($ albumin $[\mathrm{g} / \mathrm{L}] \times$ $-0.0852)$, leading to three possible grades: ALBI Score $\leq-2.60$ (ALBI grade 1), ALBI Score $>-2.60$ to $\leq-1.39$ (ALBI grade 2), and ALBI Score $>-1.39$ (ALBI grade 3). ${ }^{48}$ The ALBI grade demonstrated superior prognostic value to the Child-Pugh score in a study of patients with HCC treated with radioembolization, particularly within patients with Child-Pugh A disease. ${ }^{49}$ ALBI grade also predicts OS after surgical resection $(p<0.001)$, transarterial chemoembolization (TACE) $(\mathrm{p}<0.001)$ and sorafenib treatment $(\mathrm{p}<0.001)$, with independent prognostic value across BCLC stages, geographic regions $(\mathrm{p}<0.001),{ }^{50}$ and for cancers being treated with immunotherapy. ${ }^{50}$

\section{Diagnostic biomarkers}

Several biomarkers have been put forward to predict prognosis in HCC, yet none are currently routinely used to guide treatment decisions for patients being considered for immunotherapy. Serum alpha-fetoprotein (AFP) has been the most widely used marker to increase the suspicion for a diagnosis of HCC, and has been included in international guidelines. ${ }^{21}{ }^{51}$ However, the value of AFP as a surveillance marker remains controversial, ${ }^{52}$ and establishing a threshold value to diagnose HCC remains a challenge. ${ }^{52} 53$ Tumor-derived AFP has also been implicated in impaired dendritic cell function. ${ }^{54}$ Glypican 3 (GPC3), an antigen that is highly expressed on tumor cells and minimally present on healthy tissues, ${ }^{55}$ has been proposed as a serum biomarker for HCC and is being pursued as a target for chimeric antigen receptor (CAR) T cell therapies. ${ }^{56}$ However, neither AFP nor GPC3 have demonstrated predictive power for patients being treated with ICIs, although this is an active area of research.

The GALAD score, which determines risk of HCC based on patient sex, age, and serum levels of AFP, AFP isoform L3, and des-gamma-carboxy prothrombin has been validated for detection of HCC in patients with non-alcoholic steatohepatitis (NASH) with and without cirrhosis. ${ }^{57}$ A combination of GALAD and ultrasound (GALADUS) score has been shown to further improve performance, with an area under the curve of 0.98 (95\% CI 0.96 to 0.99 ; cut-off -0.18 ; sensitivity $95 \%$; specificity $91 \%$ ) in a single-center cohort of 111 patients with HCC and 180 controls with cirrhosis or chronic HBV. ${ }^{58}$ In March of 2020, the FDA granted breakthrough device designation to the Elecsys GALAD score to aid in early diagnosis of HCC (for further discussion of immunotherapy-specific biomarkers, including PD-L1 status, see the Patient selection and management section).

\section{Panel recommendations}

- A multidisciplinary tumor board review of liver lesions is recommended for HCC diagnosis and the development of a management plan.

- Notwithstanding that LI-RADS-5 is nearly $100 \%$ specific for HCC (LE: 1), histologic confirmation is recommended for patients with unresectable disease particularly prior to the initiation of systemic therapy. Histologic diagnosis is mandatory for non-cirrhotic patients.

- Despite the controversy regarding the scoring and staging systems that could be used, before initiation of systemic therapy, an evaluation of liver function, including aspartate transaminase (AST)/alanine aminotransferase (ALT), bilirubin, prothrombin time (PT)/international normalized ratio (INR), albumin, plus platelets, is critical (LE: 2).

- For patients being considered for immunotherapy, an HCC-specific staging system incorporating liver function assessment is suggested (LE: 2).

- To evaluate patients prior to receiving immunotherapy, Child-Pugh classification would be the most appropriate to date (LE: 1 ) to measure liver function.

\section{RECOMMENDED IMMUNOTHERAPIES FOR HCC Available agents and indications}

For more than 10 years, sorafenib was the only systemic therapy approved by the FDA for the treatment of HCC. Since 2017, four ICI regimens have entered the clinic after having received full or accelerated approval by the FDA for the treatment of advanced HCC. Only one combination, atezolizumab with bevacizumab, had full FDA approval at the time of guideline preparation, the remaining regimens (nivolumab and pembrolizumab monotherapy and nivolumab with ipilimumab) received accelerated approvals, and FDA review of confirmatory trials is ongoing. Results of the landmark trials leading to these approvals are described in table 4. Further details for each indication are discussed in chronological order of their FDA approvals. 
Table 4 Landmark trials leading to FDA approvals for immunotherapy for HCC

\begin{tabular}{|c|c|c|c|c|c|}
\hline Trial (NCT\#) & Phase & Agent(s) evaluated & Study population & Patients & Outcomes \\
\hline $\begin{array}{l}\text { CheckMate } 040 \\
\text { (NCT01658878) }\end{array}$ & I/II & Nivolumab*† & $\begin{array}{l}\text { Patients with histologically } \\
\text { confirmed advanced HCC } \\
\text { with or without HCV or } \\
\text { HBV infection. Previous } \\
\text { sorafenib treatment was } \\
\text { allowed. CP A or B7 } \\
\text { disease for dose escalation; } \\
\text { CP A disease for dose } \\
\text { expansion. }\end{array}$ & 262 & $\begin{array}{l}\text { ORR } 20 \% \\
\text { (95\% } \mathrm{Cl} 15 \% \text { to } 26 \% \text { ) in } \\
\text { dose-expansion phase } \\
\text { ORR } 14.3 \% \\
\text { (95\% } \mathrm{Cl} 6 \% \text { to } 28 \% \text { ) in } \\
\text { population with progressive } \\
\text { disease on/intolerance to } \\
\text { sorafenib }\end{array}$ \\
\hline $\begin{array}{l}\text { KEYNOTE-224 } \\
\text { (NCT02702414) }\end{array}$ & 1 & Pembrolizumab* & $\begin{array}{l}\text { Patients with disease } \\
\text { progression on or after } \\
\text { sorafenib or intolerant to } \\
\text { sorafenib, and measurable } \\
\text { CP A disease. }\end{array}$ & 104 & $\begin{array}{l}\text { ORR } 17 \% \\
\text { (95\% Cl } 11 \% \text { to } 26 \%)\end{array}$ \\
\hline IMbrave150‡ & III & $\begin{array}{l}\text { Atezolizumab+ } \\
\text { bevacizumab vs sorafenib }\end{array}$ & $\begin{array}{l}\text { Patients with unresectable } \\
\text { HCC who had received no } \\
\text { prior systemic therapy and } \\
\text { had well-compensated liver } \\
\text { disease. }\end{array}$ & 501 & $\begin{array}{l}\text { OS HR } 0.58(95 \% \text { Cl } 0.42 \\
\text { to } 0.79 ; p<0.001) \\
\text { ORR } 27.3 \% \text { vs } 11.9 \% \\
\text { (p<0.001) }\end{array}$ \\
\hline
\end{tabular}

*Accelerated approval contingent on confirmatory trials †Indication voluntarily withdrawn July 2021

¥Updated data with 12 additional months of follow-up found ORR of $29.8 \%$ (95\% $\mathrm{Cl} 24.8 \%$ to $35.0 \%$ ) for atezolizumab+bevacizumab versus $11.3 \%$ (95\% Cl $6.9 \%$ to $17.3 \%)$ for sorafenib 66

$\mathrm{Cl}$, confidence interval; CP, Child-Pugh; FDA, US Food and Drug Administration; HBV, hepatitis B virus; HCC, hepatocellular carcinoma; HCV, hepatitis $\mathrm{C}$ virus; HR, hazard ratio; ORR, overall response rate; OS, overall survival.

\section{Prior sorafenib therapy}

In 2017, nivolumab received accelerated approval as monotherapy for the treatment of patients with HCC with progression following or intolerance to sorafenib. Approval was based on data from a cohort of patients from the CheckMate 040 Trial, a phase I/II, open-label, multicenter study. Among the 154 patients treated with nivolumab, 22 (14.3\%; 95\% CI 9.2\% to $20.8 \%$ ) had an objective radiologic response based on Response Evaluation Criteria in Solid Tumors (RECIST) v1.1 criteria. Three patients (1.9\%) had complete responses (CRs) and $19(12.3 \%)$ had partial responses (PRs). DORs ranged from 3.2 months to $38.2+$ months with $91 \%$ lasting 6 months or longer and 55\% lasting 12 months or longer. The overall response rate (ORR), based on modified RECIST (mRECIST), was $18.2 \%$ (28 patients; $95 \% \mathrm{CI} 12.4 \%$ to $25.2 \%$ ) and the CR rate was $3.2 \%$ ( 5 patients) with a PR rate of $14.9 \%$ (23 patients). No differences in response rates were observed across PD-L1 expression levels. ${ }^{13}$ Postregistration studies support the safety of single-agent nivolumab in patients with ChildPugh B disease ${ }^{59}$ where treatment is associated with shorter OS compared with Child-Pugh A disease (7.3 months vs 16.3 months; $\mathrm{p}<0.001) .{ }^{60}$ Data from cohort 5 of CheckMate 040, which included 25 sorafenib-naïve and 24 sorafenib-treated patients with Child-Pugh B7-B8 advanced HCC, also showed safety and efficacy for singleagent nivolumab in a setting of mild to moderate liver impairment $^{61}$ (for further details on immunotherapy in special patient populations, see the Patient selection and management section). Continued accelerated approval for nivolumab monotherapy was contingent on the confirmatory trial CheckMate 459 (described below).

On March 10, 2020, nivolumab in combination with ipilimumab received accelerated approval by the FDA to treat patients with HCC who were previously treated with sorafenib. Approval was based on the results of an additional cohort (cohort $4^{15}$ ) from CheckMate 040. In the CheckMate 040 cohort 4, 148 patients were randomized 1:1:1 to three different treatment arms to evaluate different dosing regimens of the combination: highdose ipilimumab, low-dose ipilimumab and continuous nivolumab/ipilimumab for arms A, B, and C, respectively. For approval, efficacy was evaluated in the 49 patients who received nivolumab at $1 \mathrm{mg} / \mathrm{kg}$ and ipilimumab at $3 \mathrm{mg} / \mathrm{kg}$ every 3 weeks for 4 doses, followed by singleagent nivolumab every 2 weeks until disease progression or unacceptable toxicity. Data from all arms support anti-HCC activity of the regimen, however. The ORR reported for accelerated approval was 33\% ( $\mathrm{n}=16$; $95 \% \mathrm{CI}$ 
$20 \%$ to $48 \%$ ), with 4 CRs and 12 PRs. DORs ranged from 4.6 months to $30.5+$ months, with $31 \%$ of responses lasting 24 months or longer. ${ }^{62}$ An updated analysis at a minimum follow-up of 44 months found ORRs by blinded independent central review of $32 \%, 31 \%$, and $31 \%$ for arms A, $\mathrm{B}$, and $\mathrm{C}$, respectively. Median DORs were 17.5 months, 22.2 months, and 16.6 months for arms A, B, and C, and median OS for each arm was 22.2 months, 12.5 months, and 12.7 months, respectively. ${ }^{63}$

In CheckMate 459, a phase III trial evaluating the efficacy of nivolumab as a first-line monotherapy, the ORR was $15 \%$ in the nivolumab group and $7 \%$ in the group receiving sorafenib. Median OS was 16.4 months for nivolumab-treated patients and 14.7 months for sorafenib-treated patients (HR 0.85; 95\% CI 0.72 to 1.02; $\mathrm{p}=0.0752$ ). The difference in OS between the two groups did not meet prespecified thresholds for statistical significance (HR 0.84; $\mathrm{p}=0.0419) .{ }^{64}$ Nevertheless, nivolumab demonstrated a favorable safety profile, better response rate, improved tolerability, and better QOL outcomes when compared with sorafenib. A trend towards better survival and response rate was noted in patients with PD-L1 tumor proportion score (TPS) $\geq 1 \%$ (about 19\% of the randomized subjects) measured by the Dako PD-L1 immunohistochemistry (IHC) assay, supporting the importance of predictive biomarker development. In a 4:5 vote, the FDA Oncologic Drugs Advisory Committee (ODAC) recommended rescinding the indication for nivolumab for the treatment of patients with HCC and prior sorafenib therapy. There was unanimous agreement from committee members that voting was difficult due to the many factors, including the earlier vote to maintain the indication for pembrolizumab monotherapy. Those in favor of continued accelerated approval for nivolumab in this patient population highlighted the unmet need for second-line options. Rationale against continuing the indication centered on the lack of OS benefit in CheckMate 459 and the inadequacy of the proposed alternative studies to generate satisfactory evidence for efficacy in the second-line setting. Discussion also narrowed in on whether data exist to recommend nivolumab monotherapy over an ipilimumab plus nivolumab combination regimen, including debate over whether the group of patients deemed unfit for the dual checkpoint inhibitor combination represent a new indication that was not formally defined nor evaluated in trials to date. In July 2021, the nivolumab monotherapy indication for HCC was voluntarily withdrawn.

Accelerated approval was granted to pembrolizumab in 2018 for patients with HCG who have previously received sorafenib based on results from the phase II KEYNOTE224 Trial. The study enrolled 104 patients to receive single-agent pembrolizumab with advanced HCC and radiographic progression or intolerance to sorafenib. The ORR was $17 \%$ (95\% CI $11 \%$ to $26 \%$ ) and among the 18 patients who responded, there was $1 \mathrm{CR}$ and 17 PRs. At data cut-off, 12 of the 18 responses were ongoing and the median DOR was not reached (range 3.1-14.6+ months).
Of the responders, $89 \%$ had a DOR $\geq 6$ months, and $56 \%$ had a DOR $\geq 12$ months. ${ }^{14}$

The phase III KEYNOTE-240 confirmatory trial evaluating pembrolizumab versus placebo was negative based on the co-primary end point of median OS and PFS. Median OS was 13.9 months (95\% CI 11.6 to 16.0) for pembrolizumab versus 10.6 months (95\% CI 8.3 to 13.5 ) for placebo (HR 0.781; 95\% CI 0.611 to $0.998 ; \mathrm{p}=0.0238$ ), and median PFS for pembrolizumab was 3.0 months (95\% CI 2.8 to 4.1 ) versus 2.8 months (95\% CI 1.6 to 3.0; HR 0.718; $95 \%$ CI 0.570 to $0.904 ; \mathrm{p}=0.0022$ ), but this did not meet statistical significance by the prespecified statistical plan. ${ }^{65}$ The study did confirm the single-agent response rate of pembrolizumab in this setting with an ORR of 18.3 (95\% CI 14.0 to 23.4) and a DOR of 13.8 months (range 1.5-23.6+ months). Despite the confirmatory trial not meeting prespecified end points, when the FDA ODAC reviewed the accelerated approval in April 2021 the vote to maintain the indication for pembrolizumab was unanimous, citing unmet medical need for patients who cannot receive first-line atezolizumab with bevacizumab (described below) and who have disease progression with or become intolerant to TKIs.

\section{First-line therapy}

The first ICI regimen to receive full approval and the first to receive first-line approval for the treatment of HCC is atezolizumab in combination with bevacizumab for patients who have not received prior systemic therapy, which was approved in 2020. Approval was based on the global, open-label, phase III IMbrave150 trial, in which 501 patients with unresectable HCC were randomly assigned in a 2:1 ratio to receive either first-line atezolizumab plus bevacizumab or sorafenib monotherapy until unacceptable toxic effects or loss of clinical benefit occurred. At the primary analysis, the HR for death with atezolizumab plus bevacizumab compared with sorafenib was 0.58 (95\% CI 0.42 to $0.79 ; \mathrm{p}<0.001)$. The 12 -month OS rate was $67.2 \%$ (95\% CI $61.3 \%$ to $73.1 \%$ ) with atezolizumab plus bevacizumab and $54.6 \%$ (95\% CI $45.2 \%$ to $64.0 \%$ ) with sorafenib. Median PFS was 6.8 months (95\% CI 5.7 to 8.3 ) versus 4.3 months (95\% CI 4.0 to 5.6) with atezolizumab plus bevacizumab versus sorafenib, respectively (HR for disease progression or death $0.59 ; 95 \%$ CI 0.47 to 0.76 ; $\mathrm{p}<0.001) .{ }^{16}$ In an updated post hoc survival analyses, median OS was 19.2 months with atezolizumab plus bevacizumab compared with 13.4 months with sorafenib (HR $0.66 ; 95 \%$ CI 0.52 to $0.85 ; \mathrm{p}=0.0009$ ). The OS rates at 18 months were $52 \%$ vs $40 \%$ with atezolizumab plus bevacizumab versus sorafenib, respectively. ${ }^{66}$

The combination therapy also delayed deterioration in QOL compared with sorafenib monotherapy. In terms of the tolerability profile, grade 3-4 adverse events (AEs) occurred in $57 \%$ of patients treated with atezolizumab with bevacizumab. ${ }^{16}$ Additionally, the development of anti-drug antibodies (ADAs) is a possibility in patients treated with atezolizumab. ${ }^{67}$ In IMbrave150, among 318 ADA-evaluable patients with HCC, 30\% (n=94) tested 
positive for treatment-emergent ADAs at one or more post-dose time points. In exploratory adjusted analyses, patients who were ADA-positive at landmark week 6 had a similar OS with atezolizumab plus bevacizumab versus sorafenib, whereas those with ADA-negative status had an improved OS compared with sorafenib. However, similar PFS and ORR benefit was seen with the combination over sorafenib regardless of ADA status. ${ }^{68}$

\section{Panel recommendations}

- For first-line treatment of patients with advanced Child-Pugh A HCC, atezolizumab plus bevacizumab is recommended, unless either medication is contraindicated (LE: 2).

- General contraindications to bevacizumab include high risk of cardiac disease, stroke, hemorrhage, hemoptysis, gastrointestinal perforation, or nonhealing wounds (LE: 1). (For contraindications to immunotherapy, see the Patient selection and management section). Consideration should be given to timing of prior events. Additional contraindications specifically relevant to HCC include untreated or incompletely treated gastroesophageal varices at risk for bleeding (LE: 2).

- For patients with contraindications to atezolizumab plus bevacizumab treatment, lenvatinib or sorafenib should be considered as standard first-line therapy (LE: 2).

- Nivolumab monotherapy has demonstrated activity in Child-Pugh B7-B8 HCC for both first-line treatment of sorafenib-naïve patients and for second-line treatment of patients who were intolerant to or progressed on sorafenib (LE: 3).

- For patients with good performance status who have progressed on first-line therapy and have not received prior immunotherapy, other non FDA-approved or conditionally approved anti-PD-1 checkpoint inhibitors may be considered as immunotherapeutic options (LE: 3).

\section{IMMUNOTHERAPIES IN DEVELOPMENT FOR HCC}

The potential benefit of ICIs as monotherapies or in combination regimens including other ICIs or antiVEGF agents for advanced HCC is being evaluated in several ongoing trials. Additionally, mechanistic rationale supports the integration of ICIs with locoregional therapies for disease in early stages, and some studies have reported tolerable safety with evidence for efficacy with the combination of checkpoint blockade and liverdirected therapy. Finally, the development of novel strategies such as vaccines or adoptive cell therapies is an active area of investigation, although still in early stages at the time of publication.

\section{Checkpoint inhibitors and novel combinations}

Tremelimumab, an anti-CTLA-4 ICI, has been evaluated in a pilot trial of patients with HCC with chronic HCV infection. Among the 17 patients who were assessable for tumor response, the PR rate was $17.6 \%$ and disease control rate (DCR) was $76.4 \%$ with a median time to progression of 6.48 months (95\% CI 3.95 to 9.14). Significant drops in viral load were observed in the 20 patients who were evaluable for toxicity and viral responses, and no patients needed steroids because of severe irAEs. ${ }^{69}$ Single-agent tislelizumab (anti-PD-1), ${ }^{70}$ camrelizumab (anti-PD-1), ${ }^{71}$ and durvalumab (anti-PD-L1) ${ }^{72}$ are all also being studied in phase III trials.

Combination ICI regimens are also under investigation. The FDA has approved nivolumab at $1 \mathrm{mg} / \mathrm{kg}$ with ipilimumab at $3 \mathrm{mg} / \mathrm{kg}$ regimen as a second-line treatment option for patients with prior sorafenib exposure. ${ }^{73}$ Recently, another combination regimen, durvalumab in combination with tremelimumab, reported an ORR of up to $22.7 \%$ and a median OS of up to 18.7 months in the advanced HCC population using one single dose of tremelimumab at $300 \mathrm{mg}$, with further enhancement of response among patients with $\mathrm{CD} 8^{+} \mathrm{Ki} 67^{+}$proliferative $\mathrm{T}$ cells. ${ }^{74}$ The phase III HIMALAYA trial investigating one single dose of tremelimumab with durvalumab as first-line treatment in patients with unresectable HCC is ongoing ${ }^{75}$ and this combination regimen has been granted orphan drug designation by the FDA.

In the CheckMate 040 trial cohort $6,{ }^{76}$ the efficacy and safety profile of the triplet combination of cabozantinib, nivolumab, and ipilimumab were analyzed and compared with the cabozantinib plus nivolumab doublet. A total of 71 sorafenib-naïve or sorafenib-experienced patients with advanced HCC were randomized to either receive nivolumab $240 \mathrm{mg}$ every 2 weeks with cabozantinib $40 \mathrm{mg}$ daily $(\mathrm{n}=36)$ or nivolumab $3 \mathrm{mg} / \mathrm{kg}$ every 2 weeks with ipilimumab $1 \mathrm{mg} / \mathrm{kg}$ every 6 weeks and cabozantinib $40 \mathrm{mg}$ daily $(\mathrm{n}=35)$. Although the study was not powered to directly compare efficacy of the triplet versus doublet regimens, numerically higher response rates (29\% vs 19\%), better PFS (median 6.8 vs 5.4 months) and improved median OS (not reached vs 21.5 months; 15-month OS rates: $70 \%$ vs $64 \%$ ) were observed with the three-drug combination. Nevertheless, a higher rate of treatment-emergent AEs was also observed in the triplet arm, without the emergence of new safety signals in either treatment arms.

HCC is one of the most vascularized solid tumors and anti-angiogenic agents may complement immunotherapies. Multiple anti-angiogenic multikinase inhibitors are being evaluated in combination with checkpoint inhibitors for HCC. The combination with the most available data at the time of manuscript preparation is pembrolizumab plus lenvatinib. In Study 116, an ongoing phase Ib multicenter open-label study of lenvatinib plus pembrolizumab in 104 patients with unresectable HCC, the confirmed ORRs at data cut-off were $46.0 \%(95 \%$ CI $36.0 \%$ to $56.3 \%$ ) by mRECIST and $36.0 \%$ (95\% CI $26.6 \%$ to $46.2 \%$ ) by RECIST v1.1 with median DORs of 8.6 months (95\% CI 6.9 to not estimable [NE]) and 12.6 months (95\% CI 6.9 to NE), respectively. Median OS was 22 months and treatment-related AEs of grade 
$\geq 3$ occurred in $67 \%$ of patients. ${ }^{77}$ The ongoing phase III LEAP-002 trial is also studying the combination and enrolling patients for treatment with pembrolizumab plus lenvatinib for first-line treatment of advanced HCC. ${ }^{78}$ Other ICI/TKI combination studies include avelumab with axitinib, which led to tumor shrinkage in 15 (68.2\%) and $16(72.7 \%)$ patients and an ORR of $13.6 \%(95 \%$ CI $2.9 \%$ to $34.9 \%$ ) and $31.8 \%$ (95\% CI $13.9 \%$ to $54.9 \%$ ) by RECIST and mRECIST, respectively in one study. ${ }^{79}$ Cabozantinib is being combined with atezolizumab for patients who have not received prior systemic therapy for HCC in the phase III study COSMIC-312. ${ }^{80}$

\section{Integration with local and regional therapies}

Locoregional therapies such as TACE and drug-eluting bead TACE (DEB-TACE) may induce immunogenic cell death, thus promoting $\mathrm{CD}^{+} \mathrm{T}$ cell infiltration into the tumor microenvironment, potentially synergizing with anti-PD-(L) 1 therapy ${ }^{81}$ Doxorubicin, which has been shown to cause immunogenic cell death, ${ }^{82}$ is the most commonly administered drug during TACE and DEB-TACE, and patients undergoing chemoembolization have been shown to develop AFP-specific $\mathrm{CD}^{+}{ }^{+} \mathrm{T}$ cell responses ${ }^{83}$ as well as GPC3-specific cytotoxic T cell responses. $^{84}$

A few studies have reported tolerable safety and initial efficacy outcomes with the combination of ICIs and locoregional therapies such as TACE and radiofrequency ablation (RFA). One trial enrolled 32 patients with HCC for tremelimumab therapy at two dose levels $(3.5 \mathrm{mg}$ / $\mathrm{kg}$ and $10 \mathrm{mg} / \mathrm{kg}$ intravenous [IV]) every 4 weeks for 6 doses, followed by infusions every 3 months until offtreatment criteria were met. On day 36 , patients underwent subtotal RFA or chemoablation. Of the 19 evaluable patients, 5 (26.3\%; 95\% CI 9.1\% to $51.2 \%)$ achieved PR. The median time to tumor progression was 7.4 months (95\% CI 4.7 to 19.4) and median OS was 12.3 months (95\% CI 9.3 to 15.4$){ }^{85}$

\section{Integration with transplant}

Checkpoint inhibitors are considered contraindicated in patients undergoing transplantation due to fears of graft rejection. ${ }^{86}$ Reports have emerged of immunotherapy being used as salvage therapy in liver transplant recipients with malignancies other than HCC, but rejection was frequent. A review of 14 cases of liver transplant recipients who were treated with ICIs identified four cases of liver graft rejection and three cases with lethal outcomes. ${ }^{87}$ Another retrospective study including 39 patients with solid organ transplants reported permanent discontinuation of ICIs in $31 \%$ because of allograft rejection. Graft loss occurred in $81 \%$, leading to death in $46 \% .{ }^{88}$

\section{Vaccines}

Some vaccines have demonstrated manageable safety and preliminary efficacy in early phase trials in HCC. Although no antitumor effects or immune responses were detected among 40 patients with advanced HCC who were treated with low-dose cyclophosphamide in combination with a telomerase peptide vaccine (GV1001), ${ }^{89}$ other strategies have posted more promising results.

Several groups have attempted to develop peptide vaccines based on GPC3. One GPC3 peptide vaccine was well tolerated in a phase I trial that included 33 patients with advanced HCC. Vaccination induced a GPC3-specific cytotoxic $\mathrm{T}$ lymphocyte response in $90 \%$ of patientsthere was $1 \mathrm{PR}$ and 19 cases of stable disease at 2 months. ${ }^{90}$ That same vaccine was shown to lead to numerically lower rates of recurrence compared with surgery alone at 1 year (28.6\% vs $54.3 \%)$ and 2 years $(39.4 \%$ vs $54.5 \%)$ in the adjuvant setting in a phase II trial of 35 patients with HCC who had undergone resection. ${ }^{91}$

AFP-based vaccines have been shown to elicit $T$ cell responses in early trials. Four immunodominant, human leukocyte antigen (HLA)-A*0201-restricted epitopes of AFP that are recognized by the human $\mathrm{T}$ cell repertoire have been identified. ${ }^{92}$ In a pilot phase I clinical trial that enrolled six HLA-A*0201 patients with AFP-positive HCC for intradermal vaccinations with the four peptides emulsified in incomplete Freund's adjuvant, T cell responses were observed against most or all of the epitopes ${ }^{93}$ Subsequently, a phase I/II trial that included 10 HLA-A*0201 patients with AFP-positive HCC who were immunized with intradermal vaccinations of the four AFP peptides pulsed onto autologous dendritic cells found statistically significant levels of AFP-specific T cells to at least one peptide by major histocompatibility complex (MHC) tetramer in $60 \%$ of participants. ${ }^{94}$

Tumor lysate-based vaccines have also been evaluated in HCC. One study found that autologous tumor vaccination significantly delayed time to recurrence in 60 patients with HCC who had undergone curative resection. The 1-year, 2-year and 3-year recurrence rates in the 30 patients in the vaccine group were $16.7 \%, 29.2 \%$, and $33.3 \%$, respectively, compared with $30.8 \%, 53.8 \%$, and $61.5 \%$, respectively, in the control group. ${ }^{95}$ Another phase II trial of autologous dendritic cells pulsed with tumor lysate observed a radiologically determined DCR of $28 \%$ in 35 patients with advanced HCC. ${ }^{96}$ Hepcortespenlisimut-L, a tableted oral formulation derived from a heatinactivated pooled blood of patients with HCC and viral hepatitis, has entered phase III trials in patients with HCC and demonstrated clear improvements in ALT, AST, alkaline phosphatase, and bilirubin levels compared with placebo. ${ }^{97}$

The dramatically high efficacy rates seen with RNAbased vaccines during the COVID-19 pandemic has re-invigorated the study of RNA-vaccinology - a concept with roots in the immunotherapy discipline. ${ }^{98} 99$ RNA has been used as both a vaccine platform and an adjuvant to boost immunogenicity for HCC-specific epitopes, such as HLAA*02-restricted tumor-associated peptides. ${ }^{100} 101$

\section{Adoptive cell therapies}

To date, the most advanced clinical studies for cellular therapies in HCC are with cytokine-induced killer cells 
(CIKs), which are characterized by coexpression of CD3 and CD56 and can be generated by expanding human peripheral blood mononuclear cells in the presence of interferon-gamma (IFN $\gamma){ }^{102} 103$ One randomized phase III trial of CIKs as adjuvant therapy for patients with HCC undergoing resection demonstrated a median recurrence-free survival (RFS) of 44 months in the cell therapy group and 30 months in the control group (HR $0.63 ; 95 \%$ CI 0.43 to $0.94 ; \mathrm{p}=0.010$ by one-sided log-rank test). ${ }^{104}$ A meta-analysis of 13 phase II and phase III trials involving CIKs for HCC that included a total of 1,212 patients found that cellular therapy was associated with a significantly improved 1-year survival (OR 0.25; $95 \%$ CI 0.12 to $0.52 ; \mathrm{p}<0.001)$ and 2-year survival (OR 0.17; $95 \%$ CI 0.07 to $0.43 ; \mathrm{p}<0.001$ ), as well as a favorable DCR (OR 0.09; 95\% CI 0.04 to $0.25 ; \mathrm{p}<0.001$ ) and ORR (OR $0.21 ; 95 \%$ CI 0.13 to $0.35 ; \mathrm{p}<0.001) .{ }^{105}$

Allogenic natural killer (NK) cell-based adoptive therapies have also been evaluated in HCC. One study that included 40 patients with stage IV HCC found that NK cell therapy synergized with irreversible electroporation (IRE), leading to decreased AFP expression and higher median OS compared with IRE alone (10.1 months vs 8.9 months; $p=0.0078) .{ }^{106}$ Allogenic NK cell therapy also showed synergy with cryoablation in a study that included 61 patients with advanced HCC. After a median follow-up of 8.7 months (range 3.9-15.1 months), median PFS and DCR were higher among the 35 patients who received cryoablation plus NK cells compared with the 26 patients treated with cryoablation alone (PFS 9.1 months vs 7.6 months; $\mathrm{p}=0.0107$; DCR $85.7 \%$ vs $69.2 \%$; $<0.01) .{ }^{107}$

\section{Panel recommendations}

- Clinicians should encourage patients' participation in clinical trials.

- Future biomarker development might help to select a subgroup of patients benefitting from single-agent nivolumab treatment. Designing a biomarker strategy based on pretreatment and on-treatment tissue and blood samples to assess immune cell changes and other correlatives is critical to elucidate mechanisms of response or resistance to immunotherapy in combination with local therapy in early-stage HCC.

- Studies evaluating combinations of other immunotherapies with ICIs should be based on solid scientific rationale.

- Future randomized studies to compare local therapy alone to local therapy combined with immunotherapy are essential to assess the expected synergy and favorable treatment outcome of the combination strategy.

\section{PATIENT SELECTION AND MANAGEMENT Patient selection}

In selecting the appropriate patient for consideration of treatment with a standard immunotherapy-based approach (as opposed to within the context of a clinical trial), there are both general oncologic considerations as well as HCC-specific or liver-specific considerations. It is critical to account for the singular nature of HCC, as it generally arises in a damaged and potentially dysfunctional liver. As many as $43 \%$ of patients with HCC will die as a direct result of cirrhosis as opposed to cancer progression. ${ }^{108}$ Therefore, clinical trials needed for patients with more advanced liver function decompensation than Child-Pugh B7 are encouraged, especially when the main factor behind liver function deterioration is HCC progression rather than the underlying liver disease. Additional considerations include the patient's performance status and history of comorbidities, in particular the presence of any known autoimmune disorders. A patient's eligibility for treatment with anti-VEGF therapy-either with TKIs (eg, sorafenib) or monoclonal antibodies (eg, bevacizumab)—will also inform a treatment plan. Liver-specific factors that need to be considered include the stage of the HCC and the indication for treatment, the underlying synthetic liver function, and disease etiology and its bearing - if any-on outcome. There are also certain specific situations such as recurrence in the setting of liver transplant that need further study, as well as the role of biomarkers in predicting efficacy or toxicity. For many of these considerations, the data are varied in terms of the weight of evidence, which should be taken into account in regard to the degree to which they should influence the physician's decision.

\section{General considerations}

Clinical trials demonstrating efficacy for immunotherapy have largely been performed in patient populations who were required to have a good performance status (ie, Eastern Cooperative Oncology Group (ECOG) 0 to 1) in order to take part. This, of course, is a general and widely accepted principle of oncology trials which also applies to immunotherapy treatment, although two meta-analyses have demonstrated no significant differences in OS between patients stratified by performance status between the groups with ECOG 0 and with ECOG 1-2. ${ }^{109} 110$ The efficacy and tolerability of immunotherapy in patients with a performance status of $>2$ is largely unknown. Another population that is frequently excluded from trials and sometimes undertreated due to concerns about frailty is the elderly. Subgroup analyses from IMbrave150, however, found that the safety of atezolizumab in combination with bevacizumab was largely identical between elderly (aged $\geq 65$ years) and non-elderly (aged $<65$ years) patients. Furthermore, clinical benefit with atezolizumab in combination with bevacizumab compared with sorafenib was confirmed, with elderly patients having similarly improved OS, PFS, and ORR as non-elderly patients. ${ }^{11}$

Cardiovascular toxicity risk is a major consideration if anti-VEGF therapy is being considered as part of the treatment plan for a patient with HCC. Anti-VEGF therapies are associated with increased bleeding risk, which is an important consideration in this patient population, many of whom will have portal hypertension. Awareness 
of contraindications to anti-VEGF therapy is important, particularly as these agents become further incorporated into evolving immune-based standards of care. A recent analysis found that as many as $35 \%$ of patients with cancer receiving bevacizumab were treated despite the presence of contraindicating comorbidities. ${ }^{112}$

HCC is often diagnosed at an advanced stage in patients living with HIV, and the hepatotoxicity of highly active antiretroviral drugs may further exacerbate underlying liver damage. ${ }^{113} 114$ Historically, patients with HIV have been excluded from trials, leading to an unmet need for effective therapies in this population-a group that also has poorer outcomes in HCC, specifically, compared with HIV-negative individuals. ${ }^{115}$ Although not yet studied specifically in HCC, tolerable safety and efficacy with ICI therapy for a variety of solid tumors has been demonstrated for patients living with HIV. ${ }^{116} 117$

Patients with a history of autoimmune disorders have also historically been excluded from immunotherapy clinical trials given the mechanisms of action of immunotherapy agents and the risk of exacerbating existing autoimmunity. At present, the evidence for safety of ICIs in patients with pre-existing autoimmunity is limited to retrospective studies and case reports, ${ }^{118}$ which likely are not generalizable. Although one meta-analysis found that flares and irAEs in patients with autoimmune diseases treated with ICIs could often be managed, some events were severe and fatal. The overall incidence, however, could not be determined due to a lack of prospective studies. ${ }^{119}$ In addition, several studies have shown worse outcomes after ICI therapy among patients who were already taking steroids or immunosuppressive medication at baseline. ${ }^{120} 121$

Finally, racial and ethnic minorities have been reported to have higher rates of mortality from HCC in the USA. ${ }^{122}$ Minority groups also have a history of underrepresentation in clinical trials, ${ }^{123}$ meaning that often these patients not only often lack access to the best care for their disease but also that clinicians must extrapolate from data on the majority population for decision-making due to lack of direct evidence for efficacy. ${ }^{124}$ Awareness of historical disparities and efforts to include diverse populations in future studies is important to improve outcomes for all patients.

\section{HCC-specific considerations}

At present, the data in support of immunotherapy for HCC apply to patient populations who are not amenable to curative approaches for early-stage disease such as resection, ablation, transplantation, or locoregional approaches for intermediate-stage disease (see Immunotherapies in development for HCC section for a discussion of integration of immunotherapy with these approaches). While immunotherapy for HCG in the neoadjuvant setting cannot be recommended at this time, studies are ongoing that will evaluate the safety and feasibility of immunotherapy in the neoadjuvant or postoperative/ ablation setting. Encouraging results were reported in the final analysis of a phase II study evaluating nivolumab alone or nivolumab with ipilimumab as neoadjuvant therapy with an overall pathologic CR rate of $24 \%$ among 21 evaluable patients ( 2 patients in the nivolumab monotherapy group and 3 in the nivolumab plus ipilimumab group). Grade 3 toxicity was experienced by five patients receiving nivolumab plus ipilimumab and one receiving nivolumab monotherapy, and no grade $\geq 4$ toxicity was reported. ${ }^{125}$ The phase Ib PRIME-HCC trial will also assess safety and bioactivity of preresection nivolumab with ipilimumab in patients with HCC. ${ }^{126}$ Additionally, the combination of neoadjuvant nivolumab and cabozatinib has been evaluated in an open-label, single-arm, phase I study in patients with borderline resectable or locally advanced HCC. Among the 12 patients who underwent successful surgical resection, $41.7 \%(\mathrm{n}=5)$ had a major or complete pathologic response with $80 \%$ of the pathologic responders $(n=4)$ remaining recurrence-free at a median follow-up of 1year. Resection specimens from patients with responsive disease showed evidence for enrichment of IFN $\gamma^{+}$effector memory $\mathrm{CD} 4^{+} \mathrm{T}$ cells as well as granzyme $\mathrm{B}^{+}$effector $\mathrm{CD} 8^{+} \mathrm{T}$ cells. ${ }^{127}$

$\mathrm{HBV}$ infection is the etiological agent for as much as $50 \%$ of the incidence of HCC worldwide, ${ }^{128}$ and HCV is estimated to account for up to one-third of cases. ${ }^{129}$ HCV-associated advanced HCC was the first setting in which ICIs were evaluated, although modest response rates and a median time to progression of 6.4 months were observed in the initial study's 21-patient cohort treated with tremelimumab. ${ }^{69}$ Adequate viral control was reported in hepatitis-infected, ICI-treated patients in CheckMate 040 and KEYNOTE-224, and no worsening of hepatitis was observed. ${ }^{13}{ }^{14}$ Published trials, however, required patients with $\mathrm{HBV}$ infections to be on antiviral therapy. Another retrospective study of outcomes among immunotherapy-treated patients with concomitant HBV or HCV infections (among which HCC was the most common tumor type) found no evidence for viral reactivation and similar incidences of grade $\geq 3$ irAEs, as well as ORRs compared with those observed in registration trials of approved anti-PD-1 therapy. ${ }^{130}$ However, the immune landscape of HBV-associated HCC is generally thought to be profoundly suppressed and exhausted, which could potentially alter the efficacy of ICI therapy. A pooled analysis of anti-PD-(L) 1 therapy trials for HCC found that although HBV-positive patients achieved ORRs comparable to those of HBV-negative patients (OR 0.68 ; $95 \% \mathrm{CI}$ 0.37 to $1.25 ; \mathrm{p}=0.21$ ), the DCRs were significantly lower for HBV-positive patients compared with HBV-negative patients (OR 0.49; 95\% CI 0.27 to $0.89 ; \mathrm{p}=0.02$ ). ${ }^{131}$

A recent meta-analysis by Pfister et $a l^{132}$ found differential survival outcomes depending on HCC etiology in 1,656 patients in randomized trials of ICIs as monotherapy or in combination with bevacizumab. In the analysis, checkpoint blockade was not associated with improved survival in patients with non-viral HCC, in marked contrast to the overall cohort and patients with viral etiology. In addition, survival was also diminished in two smaller cohorts 
of patients with HCC and documented NAFLD. Although provocative and interesting, future prospective confirmatory studies are needed to understand if and how etiology affects the liver immune microenvironment. Of note, a separate study that did not include patients treated in IMbrave150 found no differences in ORRs nor features of the tumor microenvironment (TME) that are known to modulate responses to ICIs between patients with viral and non-viral HCC. ${ }^{133}$

Relatively few trials have included patients with ChildPugh B cirrhosis, a population for which few treatment options are available. In a retrospective case series of 18 patients with Child-Pugh B cirrhosis and advanced HCC who were treated with nivolumab, 94\% (17 of 18) experienced a grade $\geq 3 \mathrm{AE}$, with treatment-related grade $\geq 3$ AEs reported in $28 \%$ ( 5 of 18). IrAEs were reported in $50 \%$ of patients (9 of 18), and $28 \%$ ( 5 of 18) required steroids. Treatment-related AEs led to discontinuation of therapy in four patients $(22 \%){ }^{59}$ In the Child-Pugh B cohort of CheckMate 040, 49 patients with Child-Pugh B7 to B8 advanced HCC who were sorafenib-naïve $(n=25)$ or sorafenib-experienced $(\mathrm{n}=24)$ received nivolumab monotherapy. Investigator-assessed ORR was 12\% (95\% CI $5 \%$ to $25 \%$ ) and the DCR was $55 \%$ (95\% CI $40 \%$ to $69 \%)$. Safety was similar to that seen with nivolumab in patients with Child-Pugh A disease. At a median follow-up of 16.3 months, median OS was 7.6 months for the entire cohort-median OS in sorafenib-naïve and sorafenibtreated patients were 9.8 and 7.4 months, respectively. ${ }^{61}$ Importantly, there is no evidence to date indicating that immunotherapy causes further damage to impaired livers.

Patients with tumor invasion of the main trunk of the portal vein, invasion of the portal vein branch contralateral to the primarily involved lobe $(\mathrm{Vp} 4)$, bile duct invasion, and/or tumor occupying $\geq 50 \%$ of the liver are considered high risk. Data from IMbrave150 indicates that atezolizumab with bevacizumab is safe and effective in patients with high-risk features. Although more grade 5 upper gastrointestinal hemorrhage events were reported in high-risk patients receiving atezolizumab with bevacizumab, none of these grade 5 events were considered by investigators to be related to treatment. ${ }^{134}$ However, variceal bleeding is a potential toxicity of anti-VEGF agents. Therefore, for patients treated with atezolizumab in combination with bevacizumab, esophagogastroduodenoscopy to evaluate for varices within 6 months of initiating therapy is recommended. ${ }^{135}$

Finally, patients who have received liver transplants are typically excluded from clinical trials due to concerns about graft rejection, and high rates of rejection and mortality have been reported in the limited cases published thus far. ${ }^{87}$

\section{Biomarkers for ICI efficacy and safety in HCC}

ICIs provide benefit for only a subset of patients. The ability to identify intrinsic resistance to ICIs would allow patients to attempt other therapies, which could, most importantly, lead to better outcomes, while also saving healthcare resources. Unfortunately, validated blood or tissue biomarkers for ICI resistance are currently lacking in the clinical setting. Early studies have also returned conflicting results. High serum AFP levels are associated with increased sensitivity to the anti-VEGF receptor (VEGFR) monoclonal antibody ramucirumab. ${ }^{136}$ Post hoc subgroup analysis of randomized trials have shown that the HR for OS was slightly lower among patients with high AFP in KEYNOTE-240 (pembrolizumab vs placebo) ${ }^{65}$ and CheckMate 459 (nivolumab vs sorafenib), ${ }^{137}$ while the contrary was observed in IMbrave150 (atezolizumab with bevacizumab vs placebo). ${ }^{16}{ }^{69}$ Furthermore, objective remissions occur irrespective of AFP levels after nivolumab or pembrolizumab monotherapies, or the combination of ipilimumab and nivolumab.

A number of features of the tumor microenvironment have been associated with HCC prognosis, including overall lymphocyte infiltration, density of Tregs, and tumor-associated macrophages (TAMs), especially if M2-polarized. In melanoma, the presence of conventional type 1 dendritic cells seems critical to promote a $\mathrm{T}$ and NK cell infiltrate and for the action of ICIs. ${ }^{138} \mathrm{In}$ HCC animal models, $\beta$-catenin-mutations in HCC (which are present in around 25\% of human HCCs) result in a paucity of intratumoral conventional type 1 dendritic cells, ${ }^{139}$ and it has been proposed that $\beta$-catenin defects may be used to identify patients with disease that will fail to respond to PD-1 blockade. ${ }^{140}$ This feature awaits investigation in clinical trials. Soluble factors also modulate the immune response against HCC. For example, transforming growth factor beta (TGF- $\beta$ ) downregulates antitumor responses through a variety of different mechanisms, and high levels of the cytokine shape the response to pembrolizumab. ${ }^{141}$

Pretreatment tumor infiltration by $\mathrm{T}$ cells and their activity status are key to determine response to ICIs in various cancers. In advanced $\mathrm{HCC}, \mathrm{CD} 4^{+}$and $\mathrm{CD} 8^{+} \mathrm{T}$ cell infiltration showed weak correlations with survival after second-line treatment with PD-1 inhibitors in CheckMate $040 .{ }^{142}$ In the trial, deep antitumor responses were observed regardless of PD-L1 expression after nivolumab treatment, although the response rate was higher among patients with at least $1 \%$ of tumor cells expressing PD-L1. ${ }^{142}$

On the other hand, PD-L1 expression in tumor or stromal immune cells was higher among responders to pembrolizumab, but remissions also occurred in the absence of expression in both cell types. ${ }^{65}$ In CheckMate 459 , median OS after nivolumab and sorafenib was 16.1 months versus 8.6 months among patients that had tumor PD-L1 expression $\geq 1 \%$ (HR 0.80), and 16.7 months versus 15.2 months among those that had tumor PD-L1 expression $<1 \%$ (HR 0.84). ${ }^{137}$ Interestingly, macrophage infiltration, including M2-polarized TAMs, was not associated with clinical outcomes after nivolumab treatment. A metaanalysis including 894 patients across nine trials of ICIs in advanced HCC found a positive association between PD-L1 expression and response to therapy-especially for 
single-agent anti-PD-1. Strikingly, in the analysis, PD-L1 expression status had minimal association with response to therapy for patients being treated with anti-CTLA-4containing combinations. ${ }^{143}$ Analytical heterogeneity in PD-L1 expression is substantial, however, and may contribute to the performance of this test as a predictive biomarker. ${ }^{144}$

Several inflammatory gene signatures are correlated with higher response rate and improved OS after nivolumab treatment. ${ }^{142}$ Interestingly, the most complex transcriptomic classifications of inflammatory HCC including a large number of genes ${ }^{145}$ were not identified as predictive of response in this analysis, suggesting that short gene signatures may be more relevant for clinical development. Regarding ICI combinations, objective remissions occurred with ipilimumab plus nivolumab irrespective of PD-L1 expression in tumor cells. ${ }^{146} \mathrm{An}$ early burst of $\mathrm{Ki} 67^{+} \mathrm{CD} 8^{+}$cells in the peripheral blood was also seen in one of the randomized expansion cohorts for Study 22, which evaluated combinations of durvalumab and tremilimumab at different dosing regimens, ${ }^{74}$ hinting that cytotoxic $\mathrm{T}$ cell proliferation after therapy may predict response. Altogether, though, it seems unlikely that a single biomarker could be used to inform clinical decisions in a timely fashion. However, it is probable that composed and integrative multifactorial indexes might help identify patient subsets who are likely to benefit, further underscoring the importance of obtaining pretreatment tumor biopsies for future translational studies.

Pembrolizumab is FDA-approved for two tissueagnostic indications based on tumor-intrinsic characteristics. Approval for pembrolizumab for the treatment of microsatellite-high (MSI-H) or mismatch repair deficient (dMMR) tumors was based on a pooled ORR of $39.6 \%$ (95\% CI $31.7 \%$ to $47.9 \%$ ), with a $7 \%$ CR rate among 149 patients with 15 different tumor types in five single-arm multi-cohort multicenter trials: KEYNOTE-016, ${ }^{147}$ KEYNOTE-164, ${ }^{148}$ KEYNOTE-012 ${ }^{149}$ KEYNOTE-028, ${ }^{150}$ and KEYNOTE-158. ${ }^{151}$ Approval for pembrolizumab for non-MSI-H/dMMR tumors with high mutation burden (TMB-H) — defined as $\geq 10$ mutations per megabase $(\mathrm{mut} / \mathrm{Mb})$ as assayed by the FoundationOne CDx companion diagnostic - was based on KEYNOTE-158. ${ }^{152}$ No patients with HCC were included in the cohorts upon which the tissue-agnostic indications for pembrolizumab were approved, however.

TMB correlates with the number of neoantigens and response to ICIs in tumors with $>20$ somatic mut $/ \mathrm{Mb}$, such as melanoma. ${ }^{153} 154$ However, HCC is infrequently MSI-H/dMMR or TMB-H. One study that performed comprehensive genomic profiling of 755 patients with advanced HCC found a median TMB of 4 mut/Mb and that only six tumors $(0.8 \%)$ were TMB-H. Furthermore, out of 542 cases assessed, only one $(0.2 \%)$ was MSI-H..$^{155}$ Another analysis found a rate for MSI-H as low as $6 \% .{ }^{156}$

Markers of systemic inflammation like neutrophil to lymphocyte ratio (NLR) and platelet to lymphocyte ratio
(PLR) have shown a strong prognostic impact in HCC across tumor stages. Lower NLR has been associated with better outcomes after sorafenib, ${ }^{157} 158$ and similar trends are emerging from trials of ICIs. In CheckMate 040, patients progressing on nivolumab had a higher NLR and PLR than patients who had disease control as the best overall response. ${ }^{142}$ Consistent with this observation, a retrospective analysis of 103 patients who received nivolumab found that patients with Child-Pugh A disease who achieved PR or CR had significantly lower posttreatment NLR and PLR ( $<<0.001$ for both) compared with patients who had stable or progressive disease. ${ }^{159}$

The composition of the gut microbiota, which has been linked to the promotion of HCC development and progression through secreted metabolites, ${ }^{160}{ }^{161}$ may also predict response to treatment, although current analyses in the liver cancer setting are small and preliminary. Gut microbial diversity has been linked to ICI efficacy in epithelial tumors, ${ }^{162}$ and retrospective analysis has shown that antibiotic use is associated with worse outcomes with immunotherapy in lung and renal cancer, ${ }^{163}$ a finding that has also been replicated in a prospective trial including several additional tumor types. ${ }^{164}$ One pilot study of eight patients with HCC treated with anti-PD-1 therapy after progression on sorafenib found that patients with responsive disease displayed higher taxa richness and more gene counts in their microbiota compared with nonresponders, with enrichment for 20 distinct species of bacteria, including Akkermansia muciniphila and the Ruminococcaceae family. ${ }^{165}$ The potential for the gut microbiota to shape responses to immunotherapy is an ongoing area of research, but, at present, the state of the data is not sufficient to alter management in this regard and clinical judgment outweighs other considerations.

An additional ongoing area of research is the identification of biomarkers for the prediction of which patients will experience irAEs with ICI therapy. Several studies have reported a link between various clinical and bloodbased or serological factors and the onset of immunerelated toxicity, although none have been prospectively validated for HCC. Patients with sarcopenia ${ }^{166} 167$ and of female $\operatorname{sex}^{168169}$ have both been shown to have higher incidences of irAEs. Additionally, the composition of the gut microbiota may play a role in predicting which patients will develop ICI-associated colitis. ${ }^{170}$ Additional factors under active investigation for prediction of toxicity include elevated cytokine levels at baseline, such as interleukin (IL)- ${ }^{169}$ and IL-17, ${ }^{171}$ as well as the presence of autoantibodies. ${ }^{172} 173$ Currently there are no clinically validated biomarkers to predict the risk of irAEs.

\section{Recognition and management of irAEs}

The same mechanisms by which immunotherapy drugs exert their therapeutic effects also underlie their unique toxicities-suppression of the inhibitory mechanisms that protect tissues from uncontrolled immune responses. Unlike AEs with chemotherapy or other treatment modalities, irAEs may be delayed in onset and have prolonged 
duration, sometimes months or years after initial exposure to therapy. The overall incidence and severity of irAEs reported in phase III trials of anti-PD-(L) 1 agents varies depending on disease state and comorbidities. Most irAEs are of mild-to-moderate severity, but lifethreatening events have been reported. A meta-analysis of fatal ICI-associated toxicities encompassing more than $16,000,000$ adverse drug reactions from the medical records from the VigiBase-VigiLyze database found a total of 613 deaths related to ICIs. The fatalities related to anti-CTLA-4 therapy were most often from colitis $(n=135$, $70 \%$ ), while fatalities associated with anti-PD-(L) 1 were most often from pneumonitis $(\mathrm{n}=333,35 \%)$, hepatitis $(\mathrm{n}=115,22 \%)$, and neurotoxic effects $(\mathrm{n}=50,15 \%) .{ }^{174}$ A systematic review including 48 clinical trials involving 7,936 patients treated with nivolumab monotherapy or combination nivolumab and ipilimumab found that the double regimen was associated with more all-grade and grade $\geq 3$ irAEs categorized by system, organ, or class $(p<0.05)$. Additionally, the ORR of nivolumab combined with ipilimumab was positively correlated with the incidence rate of skin $(\mathrm{r}=0.54 ; \mathrm{p}=0.04)$ and gastrointestinal irAEs $(\mathrm{r}=0.60 ; \mathrm{p}=0.02)$, but not endocrine, hepatic, pulmonary, or renal irAEs. ${ }^{175}$ Similarly a recent observational study including 331 patients with HCC receiving anti-PD-(L) 1 monotherapy or combinations found that the emergence of treatment-related AEs of grade $\geq 2$ while on ICI therapy predicted for improved OS (median 19.7 vs 11.0 months; HR 0.32 ; $95 \%$ CI 0.16 to $0.65 ; \mathrm{p}=0.001$ ) and increased ORR ( $30 \%$ vs $\left.16 \% ; \chi^{2} 5.9 ; \mathrm{p}=0.01\right) .{ }^{176}$

Typically, the management of irAEs includes interruption of ICIs, corticosteroids, and occasionally the administration of immunomodulatory agents including tumor necrosis factor (TNF) inhibitors. Detailed recommendations on the recognition and management of ICI-associated AEs have been published elsewhere ${ }^{177}$ and the general principles contained therein may guide treatment decisions for irAEs, which are not specific to patients with HCC.

\section{IrAEs specific to the treatment of HCC}

Outside of immune-mediated hepatotoxicity, the commonly reported AEs in published trials leading to ICI approvals for HCC have been generally comparable to those seen in other disease settings. Pembrolizumab monotherapy showed a tolerable safety profile in KEYNOTE-224, with the most common irAEs of any grade being hypothyroidism $(n=8,8 \%)$ and adrenal insufficiency $(n=3,3 \%) \cdot{ }^{14}$ In the cohort of patients receiving nivolumab monotherapy in CheckMate 040, the most common AEs were pruritus $(n=9,11 \%)$ and rash $(n=11$, $23 \%) .{ }^{13}$ The addition of ipilimumab to nivolumab, as evaluated in cohort 4 of CheckMate 040, was associated with a wider variety of toxicities with the most common AEs of any grade being rash $(n=14,29 \%)$, pruritus $(n=22,45 \%)$, diarrhea $(\mathrm{n}=12,24 \%)$, decreased appetite $(\mathrm{n}=6,12 \%)$, fatigue $(n=9,18 \%)$, adrenal insufficiency $(n=7,14 \%)$, and hypothyroidism $(\mathrm{n}=10,20 \%) .{ }^{178}$ For the combination of atezolizumab with bevacizumab in IMbrave150, the most common adverse reactions were hypertension $(n=98$, $29.8 \%)$, fatigue $(n=67,20.4 \%)$, and proteinuria $(n=66$, $20.1 \%$ ), and no serious AEs with a difference in incidence of $>2 \%$ were noted between the atezolizumab with bevacizumab and sorafenib treatment groups. ${ }^{16}$

\section{Drug-induced hepatotoxicity}

HCC usually develops in a background of chronic liver disease, which itself may give rise to systemic manifestations. Cirrhosis is characterized by diffuse fibrosis of the liver, altered hepatic blood flow and portal hypertension, and progressive failure of liver functions. In parallel, other organs frequently develop secondary dysfunction. Many extrahepatic disorders associated with cirrhosis cause symptoms that may mimic irAEs and therefore lead to overdiagnosis or underdiagnosis of toxicities with immunotherapy. Late recognition of irAEs may delay treatment and worsen the prognosis. Overdiagnosis may result in inappropriate interruption of ICIs, complications caused by immunosuppressive therapy, unnecessary diagnostic procedures, and increased cost. Such disorders can also synergize in causing deteriorating organ function when irAEs occur. The most important cirrhosis-related disorders that may compromise the management of irAEs are summarized in table 5 .

Patients with HCC and underlying liver disease are at high risk for decompensation with additional insult to the organ. Some studies have found that underlying liver disease as opposed to cancer progression is the ultimate cause of death in almost half of patients with HCC. ${ }^{108}$ Elevated liver enzymes without clinical impairment in hepatic function were commonly reported in all of the trials that led to approvals of ICIs for HCC. ${ }^{13-15}$ Grade 3 or 4 elevations in liver enzymes were reported in $16 \%$ of patients in the dose-escalation arm of CheckMate 040 and in $12 \%$ of the patients in KEYNOTE-224. ${ }^{14}$ In KEYNOTE240, immune-mediated hepatitis events were seen in 10 patients $(3.6 \%)$ in the pembrolizumab group, approximately $90 \%$ of which resolved. ${ }^{65}$

No prospective trials have defined the best treatment approach for drug-induced hepatotoxicity in patients with HCC receiving immunotherapy. The package inserts for pembrolizumab, nivolumab, and atezolizumab all recommend monitoring for changes in liver function and administering corticosteroids for hepatitis followed by a taper. ICIs should also be withheld or discontinued if liver enzymes or bilirubin become elevated, with the thresholds varying depending on baseline values and the drug regimen being given. ${ }^{179}$ Exclusion of other causes of acute liver damage-including toxicities from concomitant medications, use of herbal supplements, viral hepatitis, and particularly tumor progression-is key to adequate management.

\section{Response evaluation}

Measurement of response rate in HCC has been controversial. The WHO (WHO) criteria $^{180}$ and the RECIST 
Table 5 Cirrhosis-related disorders that should be considered in the diagnostic workup of irAEs in patients with HCC (Adapted from Sangro et al, $\mathrm{J}$ Hepatol 2020) $)^{179}$

\begin{tabular}{|c|c|c|}
\hline Organ & irAE & Chronic liver disease \\
\hline Skin & $\begin{array}{l}\text { Pruritus } \\
\text { Rash } \\
\text { Erythema multiforme, } \\
\text { psoriasis, urticaria and } \\
\text { rosácea } \\
\text { Severe cutaneous adverse } \\
\text { reactions }\end{array}$ & $\begin{array}{l}\text { Pruritus } \\
\text { Skin disorders, including lichen planus, polyarteritis nodosa, } \\
\text { cryoglobulinemic vasculitis, and porphyria cutanea tarda (HCV- and } \\
\text { HBV-related) }\end{array}$ \\
\hline Liver & - Hepatitis & - Flares or viral infection \\
\hline Lung & - Pneumonitis & $\begin{array}{l}\text { - Hepatopulmonary syndrome } \\
\text { - Porto-pulmonary hypertension }\end{array}$ \\
\hline Kidney & - Nephritis & $\begin{array}{l}\text { Hepatorenal syndrome } \\
\text { Mixed cryoglobulinemia (HCV-related) } \\
\text { HBV-related nephropathy } \\
\text { IgA nephropathy }\end{array}$ \\
\hline Nervous system & $\begin{array}{l}\text { Encephalitis } \\
\text { - Aseptic meningitis } \\
\text { - Peripheral neuropathy } \\
\text { - Myasthenia gravis } \\
\text { - Guillain-Barre syndrome } \\
\text { - Autonomic neuropathy } \\
\text { - Transverse myelitis }\end{array}$ & $\begin{array}{l}\text { Porto-systemic encephalopathy (typical and atypical) } \\
\text { Viral-related peripheral neuropathy } \\
\text { - Wernicke's encephalopathy } \\
\text { - Autonomic neuropathy (HCV-related) }\end{array}$ \\
\hline
\end{tabular}

GI, gastrointestinal; HBV, hepatitis B virus; HCC, hepatocellular carcinoma; HCV, hepatitis C virus; irAE, immune-related adverse event.

guidelines $^{181}$ define standard measurement methods for converting radiology image observations into quantitative and statistically tractable frameworks for measuring changes in tumor size associated with therapy. However, assessments based solely on tumor size are misleading when applied to molecular targeted therapies and immunotherapies. For HCC in particular, poor correlation has been shown between the clinical benefits provided by sorafenib or locoregional interventional therapies and RECIST-based responses. ${ }^{182}$ Subsequently, the concept of 'viable tumor' was endorsed by the guidelines for the design of HCC clinical trials developed by AASLD ${ }^{183}$ and eventually incorporated into a formal proposal to amend standard RECIST criteria to address the unique complexity of HCC response assessment. The amended criteria were named mRECIST for HCC. ${ }^{184}$ In published trials of immunotherapy for HCC, RECIST v1.1 was used. In the immunotherapy setting, no significant differences exist between RECIST and mRECIST.

Several clinical investigations have shown that objective response measured by mRECIST predicts survival in patients treated by locoregional therapies. A meta-analysis including seven trials and 1,357 patients reported an OS HR (responders vs non-responders) of 0.39 (95\% CI 0.26 to $0.61 ; \mathrm{p}<0.0001) .{ }^{185}$ Another study found that EASL and mRECIST both outperformed the WHO criteria and 
RECIST for patients undergoing DEB-TACE. ${ }^{186}$ Recently, data from randomized trials confirmed that objective response by mRECIST predicts survival in patients with advanced-stage HCC receiving systemic therapies with TKIs, and suggested that objective response by mRECIST can be considered as a candidate surrogate end point of OS, although further research is needed to support this finding. ${ }^{187} 188$

Although late response after apparent disease progression on imaging has been reported in the context of immunotherapy for HCC, ${ }^{189}$ the overall incidence of pseudoprogression with ICI treatment is rare. Estimated rates of psuedoprogression across published studies range from $2 \%-10 \% .{ }^{190} 191$ Also rare, though possible, is a rapid acceleration in tumor growth after anti-PD-(L) 1 therapy, a phenomenon called hyperprogression. ${ }^{191} 192$ Although published evidence is limited, hyperprogression has been reported in small case series of patients with HCC treated with ICIs,${ }^{193}$ and retrospective analyses. ${ }^{194}$ Importantly, the evidence to date has only reported hyperprogression in the setting of anti-PD-(L) 1 monotherapy-it is unclear whether the addition of VEGF-directed antibodies to ICI therapy affects the likelihood of hyperprogressive disease after treatment.

\section{Panel recommendations}

- For patients with advanced-stage HCC and for patients with earlier-stage disease where liver-directed therapies are not considered appropriate or who have progressed after liver-directed therapy, the data at present supports first-line and subsequent-line ICI therapy use (LE: 2). Further studies are needed to confirm the efficacy of immunotherapy in the curative setting (neoadjuvant/adjuvant/perioperative) or in conjunction with intra-arterial therapies.

- In patients with HCC with cirrhosis, the data supports the use of immunotherapy in patients with underlying synthetic liver function consistent with wellcompensated cirrhosis, specifically Child-Pugh A (LE: 2 ). The panel recognizes, however, that some carefully selected patients with Child-Pugh B may derive benefit (LE: 3).

- Patients who have contraindications for the use of TKIs or anti-VEGF therapies (eg, cardiovascular comorbidities) may be suitable for anti-PD-1 monotherapy (LE: 1 ).

- The panel recommends against the use of immunotherapy in the post-transplant setting (LE: 4) due to the high risk of graft failure, given known mechanisms of ICIs.

- Additional studies are needed to assess the potential risks and benefits of immunotherapy in the pretransplant setting.

- The panel agrees that patients can be considered for immunotherapy treatment irrespective of hepatitis viral etiology (LE: 3), though it is strongly recommended that patients with $\mathrm{HBV}$ be on concomitant antiviral medication and adherent.
- While patients living with HIV have not been included in clinical trials to date, the panel believes that this is not an absolute contraindication to treatment with immunotherapy as long as the appropriate HIV therapy is instituted as per expert guidance (LE: 2), while further dedicated studies to assess such therapies in patients living with HIV remain critical.

- Historical disparities in access to clinical trial participation for underrepresented groups should be considered, with efforts made to support diversity, equity, and inclusion.

- The panel recommends against the use of routine testing of biomarkers for predicting immunotherapy efficacy, which, at this point, remains exploratory.

- The panel recommends against the use of routine testing of biomarkers for predicting irAEs, which, at this point, remains exploratory.

- Response assessment can be performed according to mRECIST criteria in patients receiving locoregional interventional therapies (LE: 3).

- Limited data are available concerning the value of mRECIST and immune-related RECIST (irRECIST) criteria in the setting of HCC response assessment, especially in the context of ICI therapy. Further studies are needed to compare outcomes between patients with response to treatment by mRECIST versus irRECIST.

- Pseudoprogression, while a real phenomenon, occurs rarely (LE: 4). A comprehensive assessment is encouraged. In published trials, treatment beyond progression has been allowed.

- Hyperprogression may occur (LE: 4). It is uncommon, cannot be anticipated, and remains poorly understood.

- Caution should be exercised in translating response assessment models developed for clinical trials into clinical practice.

- For management of irAEs in patients with HCC, refer to general principles in published guidelines.

\section{PATIENT SUPPORT AND QOL}

Immunotherapies and targeted therapies have extended survival for patients with HCC, but these new agents are not curative in most cases, and their unique toxicities can affect QOL. The importance of QOL as an independent prognostic factor for response to treatment or predicting disease progression is becoming more appreciatedseveral studies have demonstrated associations between baseline patient-reported QOL and survival in HCC. ${ }^{195-197}$ Therefore, immunotherapy treatment plans should take patient QOL at baseline and on therapy into account. Additionally, it is important for clinicians to provide patients with necessary and sufficient information to help them navigate treatment without undue emotional or financial distress. Referral to support groups is also highly encouraged, including the American Liver Foundation, Blue Faery: The Adrienne Wilson Liver Cancer Association, Cancer Support Community, the Fatty Liver Foundation, and the Global Liver Institute. In addition, 
information provided by the National Cancer Institute and SITC may be helpful for patients.

\section{Patient and caregiver education}

Prior to diagnosis, the majority of patients and their caregivers will likely be unfamiliar with HCC, and they may harbor misconceptions about the etiology of the disease, potentially leading to stigma and shame over and above the emotional distress associated with a cancer diagnosis. ${ }^{198}$ Among different types of cancer, HCC has been found to rank third highest in terms of levels of emotional distress experienced by patients. ${ }^{199}$ Rehabilitation, palliative care, and psycho-oncology have been insufficiently studied in liver cancer.

Perceived stigma surrounding liver disease may cause patients to delay care or avoid seeking social support, which negatively impacts QOL. ${ }^{200}$ The majority of HCC cases worldwide are secondary to HBV or HCV infection, ${ }^{4}$ with NASH increasingly becoming the primary cause in the US and Europe. ${ }^{5}$ However, a survey of HCC caregivers in the US found that $72 \%$ were under the mistaken impression that heavy alcohol use was the most common risk factor for liver cancer. ${ }^{201}$ Stigma surrounding HBV may be more pronounced in certain populations, such as people of Asian descent, ${ }^{202}{ }^{203}$ so it is important for healthcare providers to be sensitive and culturally informed in their communications with patients.

HCC is a disease within a disease, and patients as well as their caregivers need to understand that their treatment journey will involve both the cancer itself as well as underlying liver damage. Patients with HCC often receive care from a multidisciplinary team that may include oncologists, hepatologists, surgeons, gastroenterologists, endocrinologists, and other specialists. In addition to the care team responsible for administering therapy targeting the tumor and the liver, patients will need 'whole-person' support for psychosocial and spiritual concerns, especially during end-of-life care. ${ }^{204} 205$ Depending on the stage of their disease (for more details on staging systems for HCC, see the Diagnostics and staging for patients with HCC section), a patient may be receiving information from a large number of different providers, especially in cases of intermediate-stage HCC. ${ }^{205}$ Additionally, practitioners from other specialties may have limited knowledge about the unique mechanisms of action of immunotherapies, and the accompanying potential for toxicities, making ongoing communication between a patient and their treating oncologist paramount.

Currently, immunotherapy is only approved for patients with advanced disease. Patients may be unfamiliar with the stages of liver cancer and the difference between treatments with curative intent and palliative therapy. Further complicating matters, patients may have preconceived notions shaped by media portrayals of high-profile immunotherapy success stories, while being less knowledgeable about the realistic efficacy and potential toxicities with treatment. ${ }^{206}$ Early referral to palliative care has been shown to improve QOL in patients with non-small cell lung cancer ${ }^{207}$ yet palliative care is underutilized in patients with end-stage liver disease. ${ }^{208} 209$ Patients with cirrhosis who are ineligible for transplant are also underserved with appropriate palliative care. ${ }^{210}$ It is important for patients to understand that immunotherapy for HCC, even if it may extend OS, is a palliative treatment used in advanced stages of the disease and not curative in intent, so that they may be referred to advanced care planning early on in their treatment.

\section{Considerations for administration, dosing and monitoring}

The tolerability of immunotherapy is, for the most part, better than conventional cancer treatments, although future combination strategies (eg, ICIs with TKIs) may be associated with less favorable toxicity profiles. ${ }^{211}$ The administration, dosing, and monitoring considerations for immunotherapy may be distinct from what a patient or caregiver is expecting based on experience with prior therapies or conversations with other healthcare providers who do not specialize in immunotherapy. Therefore, it is important to discuss the potential for irAEs and the signs and symptoms of expected toxicities with patients and caregivers. Additionally, patients must understand how liver comorbidities may affect the efficacy of immunotherapy for HCC (see the Patient selection and management section for considerations for healthcare providers). It is important for patients and caregivers to have clear and detailed instructions for when to contact their healthcare providers due to symptoms of irAEs, and examples of call parameters are provided in box 1 .

\section{Box 1 Patient and caregiver education for call parameters for irAEs}

You should contact your healthcare providers for any of the following symptoms (or call 911 or seek emergency services as indicated)*:

- Abdominal pain

- Change in stool (blood or mucus in stool, change in color, light or clay colored)

- Increase in bowel movements, $>3$ movements above a patient's baseline

- Diarrhea, $>3$ watery stools

- Nausea or vomiting

- Jaundice (yellowish skin color)

- Difficulty breathing, shortness of breath, or chest tightness

- New non-productive dry cough

- Mental status changes

- New visual disturbances

- Headache

- New or worsening fatigue

- Fever with temperature $>100.4^{\circ} \mathrm{F}\left(38^{\circ} \mathrm{C}\right)$

- New weakness, muscle or joint pains

- Unintentional weight loss $>3 \mathrm{lbs}(1.5 \mathrm{~kg})$

- Significant weight gain with obvious abdominal swelling

- Rash which may or may not be accompanied by tenderness or itching

*Note to providers: Call parameters for patients highlight the following conditions: colitis, pneumonitis, endocrinopathies, dermatologic toxicities. It should be noted that many conditions have overlapping symptoms. 
The ICIs that are currently approved for HCC are typically given as IV infusions, whereas TKIs such as sorafenib are oral medications. Because immunotherapy is usually administered at an infusion center, access to care may be a challenge for some patients, especially those in rural areas. ${ }^{212}{ }^{213}$ However, a potential benefit of the requirement for inperson infusions is the opportunity for contact with a treating physician if AEs do occur. Additionally, although patients receiving palliative chemotherapy have been found to prefer oral administration over IV, the majority are not willing to accept a decreased response rate or shorter DOR, ${ }^{214}$ which is likely also true when deciding between IV immunotherapy versus other oral medications.

Multiple liver-specific assessment instruments have been developed to monitor QOL in patients with HCC, including the European Organization for Research and Treatment of Cancer Quality of Life Questionnaire Core-18 (EORTC QLQ-HCC18), ${ }^{215}$ the Functional Assessment of Cancer Therapy-Hepatobiliary (FACT-Hep), ${ }^{216}$ the FACT Hepatobiliary Symptom Index (FHSI), ${ }^{217}$ and the QOL-liver cancer (QOL-LC). ${ }^{218}$ However, multiple systematic reviews have found that the most used assessment tool is the EORTC Quality of Life Questionnaire Core-30 (EORTC QLQ-C30). ${ }^{219} 220$

\section{Special considerations for patients with HCC}

Patients with HCC have been found to have lower healthrelated QOL (HRQOL) than the general population, especially for measures of physical, psychological, and functional well-being, as well as hepatobiliary symptoms. ${ }^{219}$ Both physical and psychological factors may influence a patient's QOL, and a person's self-perception and coping mechanisms may modulate their status. In patient interviews, HCC has been found to be perceived as a longterm and chronic disease that cannot be cured but might be controlled, and coping strategies can include focusing as much as possible on managing HCC and its symptoms, emotional responses, and leading a normal life. ${ }^{221}$ Those mental constructs can affect feelings about physical symptoms, and it has been demonstrated that patients with negative illness perceptions who use more emotionoriented coping had worse HRQOL. ${ }^{222}$ However, rigorous studies on interventions targeting disease perception or coping mechanisms are currently lacking.

Pain, particularly upper quadrant abdominal pain, is common in patients with HCC. ${ }^{223}$ Pain management may be difficult because approximately $80 \%$ of patients with HCC have cirrhosis, ${ }^{224}$ and liver damage can alter drug pharmacokinetics. Perhaps due to confusion about efficacy and safety for opioid and non-opioid analgesics, patients with HCC are undertreated for pain. ${ }^{225}$ However, some generally safe options for pain management for patients with impaired liver function exist, including opioids, non-steroidal anti-inflammatory drugs (NSAIDs) in some cases, topical lidocaine patches (which have low levels of systemic absorption) for localized analgesia, tricyclic antidepressants, and anticonvulsants such as gabapentin (which is not metabolized by the liver). ${ }^{226}$

Financial toxicity is a major concern for patients with cancer, ${ }^{227}$ and immunotherapies are among the most expensive agents on the pharmaceutical market. ${ }^{228} 229$ Patients with cancer shoulder the burden of an increasing number of out-of-pocket costs for their treatment, even if they have insurance coverage. ${ }^{230}$ Treatment may cause both material and psychological financial hardship, and the risk factors for each vary. Patients of younger age, female sex, non-white race, and who change employment because of cancer are more likely to experience material financial hardship, whereas psychological hardship is more likely among those who are uninsured or have lower family income. ${ }^{231}$ The degree to which cancer causes financial burden has been shown to be the single most important predictor for poor QOL, ${ }^{232}$ and healthcare costs for HCC are substantial. In both North America and Asia, costs are highest for patients with HCC in the terminal phase of care. ${ }^{233}$ Although a comprehensive analysis of the healthcare costs associated with immunotherapy in the HCC setting has not yet been performed, oncologists should communicate with patients about how treatment may affect their financial well-being, as health insurance may not cover the costs of immunotherapy drugs.

Importantly, however, immunotherapy has generally been associated with favorable QOL outcomes compared with previous standards of care. In the landmark trials leading to FDA approval of checkpoint inhibitors for HCC, no adverse effects on QOL were observed when outcomes were reported for the patients receiving immunotherapy. Nivolumab was associated with stable patientreported outcomes, including indicators of health status and QOL regardless of prior sorafenib in CheckMate $040 .{ }^{13}$ It is noteworthy to mention that even in a subcohort of patients from the CheckMate 040 trial with impaired liver function (Child-Pugh B), the AE profile was comparable to what was seen in patients with Child-Pugh A disease. ${ }^{59}$ Additionally, IMbrave 150 provided a large and rich data set on patient-reported QOL outcomes, which complemented the efficacy data, with a reporting rate of greater than $90 \%$. The study found that fewer patients treated with the combination of atezolizumab with bevacizumab experienced QOL deterioration compared with those receiving sorafenib. Furthermore, for the patients who did experience QOL deterioration on immunotherapy, the onset was later. ${ }^{234}$ Pembrolizumab also was shown to preserve HRQOL in a prespecified exploratory analysis of patients enrolled in KEYNOTE-240. Among the 271 and 127 patients randomly assigned to pembrolizumab and placebo, respectively, who completed the EORTC QLQ-C30 and the HCC supplement EORTC QLQ-HCC18, changes in both scores were similar across arms and global health status/QOL scores were stable. ${ }^{235}$ It will be important to prospectively study QOL outcomes in future immunotherapy trials, especially as new combination regimens advance through clinical development. 


\section{Panel recommendations}

- Patient and caregiver education for HCC should include an overview of the liver's function in the body, an explanation of underlying liver diseases such as HBV, HCV, and NASH, and a discussion of how immunotherapy works to treat their cancer.

- Patients must know which provider is coordinating their treatment, and they need to have clear instructions to promptly report any signs or symptoms of potential immune-related toxicities.

- Patients need counseling on the goals of treatment in advanced HCC, which is not curative in most patients, despite significant advances. Management of HCC should include focus on supportive care for uncontrolled symptoms and inclusion of palliative care specialists.

- Patients should receive education on the expected toxicities associated with immunotherapies, including hepatitis, colitis, pneumonitis, and immune-related endocrinopathies. Detailed call parameters should be provided to promptly report signs and symptoms of irAEs.

- Assessment of patients' physical function and symptoms should be performed before, during, and after therapy.

- Patients should be referred to a treatment team including a social worker and a financial manager to assist in navigating healthcare costs and identifying support systems.

- Conversations should be initiated with patients about how the costs of immunotherapy treatment will be covered, including contributions from private insurance, Medicare and Medicaid, clinical trials, patient assistance programs, or compassionate use as needed.

- Patients should be provided information about local advocacy and support groups specific to primary liver cancer.

\section{CONCLUSION}

Immunotherapy represents a major breakthrough for the treatment of advanced HCC, offering some of the first demonstrated improvements for patient outcomes over standard-of-care systemic therapies since the late 2000s. Despite these advances, immunotherapy for HCC is currently only applicable to patients with advanced-stage disease and largely not curative in intent. Furthermore, the question of how to manage disease that progresses after ICI therapy remains unanswered. Additionally, the use of immunotherapy for early-stage disease remains largely investigational. As additional trials continue to report results, more options may become available for later lines of therapy. Future trials are needed to address the impact of immunotherapy in combination strategies with locoregional approaches, to assist oncologists and their patients in balancing the potential for harm and benefit in early-stage cancer. In the future, the indications for existing therapies are likely to continue to expand and novel combinations may be approved.
These guidelines will be updated as the field continues to develop.

\section{Author affiliations}

${ }^{1}$ Thoracic and Gl Malignancies Branch, National Cancer Institute, Bethesda, Maryland, USA

${ }^{2}$ Memorial Sloan Kettering Cancer Center, New York, New York, USA

${ }^{3}$ Weill Medical College at Cornell University, New York, New York, USA

${ }^{4}$ Department of Medical Oncology, National Taiwan University Cancer Center and

National Taiwan University Hospital, Taipei, Taiwan

${ }^{5}$ The Mater Hospital/University College Dublin, Dublin, Ireland

${ }^{6}$ Norris Comprehensive Cancer Center, University of Southern California, Los Angeles, California, USA

${ }^{7}$ David Geffen School of Medicine, University of California Los Angeles, Los Angeles, California, USA

${ }^{8}$ University of Mainz Medical Center, Mainz, Germany

${ }^{9}$ Harvard Medical School, Massachusetts General Hospital, Boston, Massachusetts, USA

${ }^{10}$ Lombardi Comprehensive Cancer Center, Georgetown University Medical Center, Washington, District of Columbia, USA

${ }^{11}$ Department of Gastrointestinal Medical Oncology, Division of Cancer Medicine,

The University of Texas MD Anderson Cancer Center, Houston, Texas, USA

${ }^{12}$ Department of Medicine (Hematology/Oncology), UCSF Helen Diller Family Comprehensive Cancer Center, San Francisco, California, USA

${ }^{13}$ Department of Radiology, University of Pisa School of Medicine, Pisa, Italy

${ }^{14}$ Miami Cancer Institute, Miami, Florida, USA

${ }^{15}$ Oncological Sciences Department, Tisch Cancer Institute, Icahn School of Medicine at Mount Sinai, New York, New York, USA

${ }^{16}$ Department of Surgery \& Cancer, Imperial College London, London, UK

${ }^{17}$ Clinica Universidad de Navarra-Instituto de Investigación Sanitaria de Navarra (IDISNA), Pamplona, Spain

${ }^{18}$ Centro de Investigación Biomédica en Red de Enfermedades Hepáticas y Digestivas (CIBEREHD), Madrid, Spain

${ }^{19}$ Federico II University Naples, Naples, Italy

${ }^{20}$ Blue Faery: The Adrienne Wilson Liver Cancer Association, Birmingham, Alabama, USA

${ }^{21}$ Queen Mary Hospital, The University of Hong Kong, Hong Kong, Hong Kong

${ }^{22}$ Jiahui Health, Jiahui International Cancer Center, Shanghai, China

${ }^{23}$ Foundation for Applied Medical Research (FIMA), Pamplona, Spain

${ }^{24}$ Centro de Investigación Biomédica en Red Cáncer (CIBERONC), Madrid, Spain

Twitter Ghassan K Abou-Alfa @GABOUALFA, Aiwu Ruth He @ruthhe12 and Andrea Wilson Woods @bluefaeryliver

Acknowledgements The authors thank the SITC staff for their contributions including Sam Million-Weaver, PhD, for medical writing; Angela Kilbert and Emily Gronseth, PhD, for editorial support, and Lionel Lim for project management and assistance. The authors also thank SITC for supporting the manuscript development.

Contributors All authors served on the SITC HCC Immunotherapy Guideline Expert Panel, drafted content, and provided critical review during the manuscript development. TFG and IM provided leadership as Chairs of the Expert Panel and provided guidance on the manuscript structure and content and thus are first and last authors; all other authors are listed alphabetically by last name. AWW was the patient advocate representative.

Funding The authors have not declared a specific grant for this research from any funding agency in the public, commercial or not-for-profit sectors.

Competing interests GKA-A-Consulting fees: Agios, AstraZeneca, Autem, Bayer, Beigene, Berry Genomics, Celgene, Debio, Eisai, Eli Lilly, Flatiron, Genentech, Gilead, Incyte, Ipsen, LAM, Merck, MINA, QED, Redhill; Contracted research: Agios, AstraZeneca, Bayer, Berry Genomics, Bristol-Myers Squibb, Casi, Exelixis, Genoscience, Incyte, Polaris, Puma, QED, Sillajen; IP rights: ARTICLES AND METHODS FOR PREVENTING AND TREATING DERMATOLOGIC ADVERSE EVENTS, identified by International Patent Application No. PCT/US2014/031545 filed on March 24, 2014, and priority application Serial No.: 61/804,907 filed on March 25, 2013; Partner consulting fees: Celgene, CytomX, Loxo, Merck, Silenseed, Sobi, Twoxar; Partner contracted research: ActaBiologica, AstraZeneca, Bristol-Myers Squibb, Celgene, Genentech, Halozyme, Mabvax, Roche, Silenseed. A-LCConsulting fees: AstraZeneca, Bristol-Myers Squibb, Eisai, Merck Serono, Novartis, 
Ono Pharmaceutical, Exelixis, Nucleix, IPSEN Innovation, Bayer Healthcare, Merck Sharp Dohme. AGD —Consulting fees: AstraZeneca. ABE-K —Consulting fees: Bayer, Bristol-Myers Squibb, ElSAl, Merck, Exelixis, Roche/Genentech, Agenus, Gilead, AstraZeneca, Target Pharma Solutions; Contracted research: AstraZeneca, Astex; Non-CME services: Bayer, Bristol-Myers Squibb, EISAl, Merck, Exelixis, Roche/ Genentech. RSF-Consulting fees: AstraZeneca, Bayer, Eisai, CStone, BristolMyers Squibb, Eli Lilly, Exilexis, Merck, Pfizer, Roche/ Genentech; Contracted research: UCLA, Eisai, Merck, Bristol-Myers Squibb, Roche/ Genentech, Pfizer, Eli Lilly. PRG—Consulting fees: Bayer, Bristol-Myers Squibb, AstraZeneca, Sirtex, MSD, Eisai, Ipsen, Roche, Lilly, Adaptimmune; Contracted research: Bayer. TFGContracted research: Astra Zeneca, Bristol-Myers Squibb, Merck, Sillajen, Vascular Biogenics,. LG—Consulting fees: Agios, Debiopharm, Taiho, Alentis, Incyte, Klus, Pieris, QED, SIRTEX, AstraZeneca, H3Biomedicine. ARH—Consulting fees: Merck, Bayer, Bristol-Myers Squibb, AstraZeneca; Contracted research: Merck, Genentech; Non-CME services: Eisai, Bristol-Myers Squibb, Exelixis. AOK —Consulting fees: Bristol-Myers Squibb, Roche/Genentech, Exelixis, Eisai; Contracted research: Bristol-Myers Squibb, Roche/Genentech, Exelixis, Eisai, Merck, Henguri, Immatics, AdaptImmune, Abivax. RKK —Consulting fees: Genentech/Roche, Gilead; Contracted research: Adaptimmune, Agios, AstraZeneca, Bayer, Bristol-Myers Squibb, Eli Lilly, EMD Serono, Exelixis, Merck, Novartis, Partner Therapeutic, QED, Taiho. RLConsulting fees: AstraZeneca, Roche, Celsion, Guerbet. AL-Contracted research: Pfizer, Genentech (collaboration grants). IM-Consulting fees: Bristol-Myers Squibb F-STAR, Alligator, Pharma Mar, AstraZeneca, Numab Therapeutics, Roche, Amunix, Gossamer, Molecular Partners, Merck-Serono, Genmab, PharmaMar; Contracted research: Roche, Bristol-Myers Squibb, Highlight Therapeutics, Alligator, Genmab, AstraZeneca. DJP—Consulting fees: ViiV Healthcare, Bayer, Hoffman La Roche, EISAl, H3B, MiNa Alpha Therapeutics, DaVolterra; Non-CME services: Hoffmann La Roche, EISAl; Contracted research: Merck Sharpe and Dohme, Bristol Myers Squibb (to institution). BS - Consulting fees: Adaptimmune, AstraZeneca, BristolMyers-Squibb, H3B Biomedicine, Ipsen, Lilly, Roche, Sirtex; Contracted research: Bristol-Myers Squibb, Sirtex Medical. AWW —Consulting fees: Eisai, Genentech; IP Rights: Cancer U; Salary: Blue Faery: The Adrienne Wilson Liver Cancer Association. TY—Consulting or advisory role: Bristol Myers-Squibb, MSD, Exelixis, Ipsen, Eisai, AstraZeneca, Bayer, Novartis, EMD Sereon, Abbvie, Pfizer, Ei Lilly, Sirtex, Sillajen, Taiho, OrigiMed, New B Innovation, Sirtex, H3 Biomedicine; Honoraria: Bristol Myers-Squibb, MSD, Exelixis, Ipsen, Eisai, AstraZeneca, Bayer, Novartis, EMD Sereon, Abbvie, Pfizer, Ei Lilly, Sirtex, Sillajen, Taiho, OrigiMed, New B Innovation, Sirtex, H3 Biomedicine. AZ - Consulting fees: Merck, Lilly, Eisai, Exelixis, Roche, Sanofi, Bayer. DMH, RIT—Nothing to disclose. SITC Staff: SMW-Shares owned: Pacific Biosciences of California Inc., Editas Medicine. EG, AK, LL—Nothing to disclose.

\section{Patient consent for publication Not required}

Provenance and peer review Not commissioned; externally peer reviewed.

Open access This is an open access article distributed in accordance with the Creative Commons Attribution Non Commercial (CC BY-NC 4.0) license, which permits others to distribute, remix, adapt, build upon this work non-commercially, and license their derivative works on different terms, provided the original work is properly cited, appropriate credit is given, any changes made indicated, and the use is non-commercial. See http://creativecommons.org/licenses/by-nc/4.0/.

\section{ORCID iDs}

Tim F Greten http://orcid.org/000-0002-0806-2535

David J Pinato http://orcid.org/0000-0002-3529-0103

Ignacio Melero http://orcid.org/0000-0002-1360-348X

\section{REFERENCES}

1 Bray F, Ferlay J, Soerjomataram I, et al. Global cancer statistics 2018: GLOBOCAN estimates of incidence and mortality worldwide for 36 cancers in 185 countries. CA Cancer J Clin 2018;68:394-424.

2 Siegel RL, Miller KD, Fuchs HE, et al. Cancer statistics, 2021. CA Cancer J Clin 2021;71:7-33.

3 Ryerson AB, Eheman CR, Altekruse SF, et al. Annual report to the nation on the status of cancer, 1975-2012, featuring the increasing incidence of liver cancer. Cancer 2016;122:1312-37.

4 El-Serag HB. Epidemiology of viral hepatitis and hepatocellular carcinoma. Gastroenterology 2012;142:1264-73.

5 Bugianesi E. Non-Alcoholic steatohepatitis and cancer. Clin Liver Dis 2007;11:191-207.
6 Bruix J, Sherman M. American association for the study of liver D. management of hepatocellular carcinoma: an update. Hepatology 2011;53:1020-2.

7 Marrero JA, Kulik LM, Sirlin CB, et al. Diagnosis, staging, and management of hepatocellular carcinoma: 2018 practice guidance by the American association for the study of liver diseases. Hepatology 2018;68:723-50.

8 Likhitsup A, Razumilava N, Parikh ND. Treatment for advanced hepatocellular carcinoma: current standard and the future. Clin Liver Dis 2019;13:13-19.

9 Wrzesinski SH, Taddei TH, Strazzabosco M. Systemic therapy in hepatocellular carcinoma. Clin Liver Dis 2011;15:423-41.

10 Bruix J, Qin S, Merle P, et al. Regorafenib for patients with hepatocellular carcinoma who progressed on sorafenib treatment (RESORCE): a randomised, double-blind, placebo-controlled, phase 3 trial. Lancet 2017;389:56-66.

11 Kudo M, Finn RS, Qin S, et al. Lenvatinib versus sorafenib in first-line treatment of patients with unresectable hepatocellular carcinoma: a randomised phase 3 non-inferiority trial. Lancet 2018;391:1163-73.

12 Llovet JM, Montal R, Sia D, et al. Molecular therapies and precision medicine for hepatocellular carcinoma. Nat Rev Clin Oncol 2018;15:599-616.

13 El-Khoueiry AB, Sangro B, Yau T, et al. Nivolumab in patients with advanced hepatocellular carcinoma (CheckMate 040): an openlabel, non-comparative, phase 1/2 dose escalation and expansion trial. Lancet 2017;389:2492-502.

14 Zhu AX, Finn RS, Edeline J, et al. Pembrolizumab in patients with advanced hepatocellular carcinoma previously treated with sorafenib (KEYNOTE-224): a non-randomised, open-label phase 2 trial. Lancet Oncol 2018;19:940-52.

15 Yau T, Kang Y-K, Kim T-Y, et al. Nivolumab (NIVO) + ipilimumab (IPI) combination therapy in patients (PTS) with advanced hepatocellular carcinoma (aHCC): results from CheckMate 040. JCO 2019;37:4012.

16 Finn RS, Qin S, Ikeda M, et al. Atezolizumab plus bevacizumab in unresectable hepatocellular carcinoma. N Engl J Med 2020;382:1894-905.

17 Keenan BP, Fong L, Kelley RK. Immunotherapy in hepatocellular carcinoma: the complex interface between inflammation, fibrosis and the immune response. J Immunother Cancer 2019;7:267.

18 Greten TF, Sangro B. Targets for immunotherapy of liver cancer. Journal of Hepatology 2018;68:157-66.

19 Graham R, Mancher M, et al, Institute of Medicine Committee on Standards for Developing Trustworthy Clinical Practice Guidelines. Clinical practice guidelines we can trust. US: National Academies Press, 2011.

20 Marrero JA, Ahn J, Rajender Reddy K, et al. Acg clinical guideline: the diagnosis and management of focal liver lesions. Am J Gastroenterol 2014;109:1328-47.

21 Vogel A, Cervantes A, Chau I, et al. Hepatocellular carcinoma: ESMO clinical practice guidelines for diagnosis, treatment and follow-up. Ann Oncol 2018;29:iv238-55.

22 European Association for the Study of the Liver. Electronic address: easloffice@easloffice.eu, European Association for the Study of the Liver. EASL clinical practice guidelines: management of hepatocellular carcinoma. J Hepatol 2018;69:182-236.

23 Kokudo N, Takemura N, Hasegawa K, et al. Clinical practice guidelines for hepatocellular carcinoma: the Japan Society of hepatology 2017 (4th JSH-HCC guidelines) 2019 update. Hepatol Res 2019;49:1109-13.

24 Chernyak V, Fowler KJ, Kamaya A, et al. Liver imaging reporting and data system (LI-RADS) version 2018: imaging of hepatocellular carcinoma in at-risk patients. Radiology 2018;289:816-30.

25 Kamath A, Roudenko A, Hecht E, et al. CT/MR LI-RADS 2018: clinical implications and management recommendations. Abdom Radiol 2019;44:1306-22.

26 Hanna RF, Miloushev VZ, Tang A, et al. Comparative 13-year meta-analysis of the sensitivity and positive predictive value of ultrasound, CT, and MRI for detecting hepatocellular carcinoma Abdom Radiol 2016;41:71-90.

27 Lee YJ, Lee JM, Lee JS, et al. Hepatocellular carcinoma: diagnostic performance of multidetector CT and Mr imaging-a systematic review and meta-analysis. Radiology 2015;275:97-109.

28 Ye F, Liu J, Ouyang H. Gadolinium Ethoxybenzyl Diethylenetriamine Pentaacetic Acid (Gd-EOB-DTPA)-Enhanced Magnetic Resonance Imaging and Multidetector-Row Computed Tomography for the Diagnosis of Hepatocellular Carcinoma: A Systematic Review and Meta-analysis. Medicine 2015;94:e1157.

29 Naugler WE, Alsina AE, Frenette CT, et al. Building the multidisciplinary team for management of patients with 
hepatocellular carcinoma. Clin Gastroenterol Hepatol 2015;13:827-35.

30 Silva MA, Hegab B, Hyde C, et al. Needle track seeding following biopsy of liver lesions in the diagnosis of hepatocellular cancer: a systematic review and meta-analysis. Gut 2008;57:1592-6.

31 Abu-Zeinah GF, Weisman P, Ganesh K, et al. Acute myeloid leukemia masquerading as hepatocellular carcinoma. J Gastrointest Oncol 2016;7:E31-5.

32 Brierley JD, Gospodarowicz MK, Wittekind C. TNM classification of malignant tumours. John Wiley \& Sons, 2017.

33 Llovet JM, Fuster J, Bruix J, et al. The Barcelona approach: diagnosis, staging, and treatment of hepatocellular carcinoma. Liver Transp/ 2004;10:S115-20.

34 A new prognostic system for hepatocellular carcinoma: a retrospective study of 435 patients: the cancer of the liver Italian program (clip) Investigators. Hepatology 1998;28:751-5.

35 Kudo M, Chung H, Osaki Y. Prognostic staging system for hepatocellular carcinoma (clip score): its value and limitations, and a proposal for a new staging system, the Japan integrated staging score (JIS score). J Gastroenterol 2003;38:207-15.

36 Leung TWT, Tang AMY, Zee B, et al. Construction of the Chinese university prognostic index for hepatocellular carcinoma and comparison with the TNM staging system, the Okuda staging system, and the cancer of the liver Italian program staging system: a study based on 926 patients. Cancer 2002;94:1760-9.

37 Chevret S, Trinchet JC, Mathieu D, et al. A new prognostic classification for predicting survival in patients with hepatocellular carcinoma. Groupe d'Etude et de Traitement Du Carcinome Hépatocellulaire. J Hepatol 1999;31:133-41.

38 Kim BK, Kim SU, Park JY, et al. Applicability of BCLC stage for prognostic stratification in comparison with other staging systems: single centre experience from long-term clinical outcomes of 1717 treatment-naïve patients with hepatocellular carcinoma. Liver Int 2012;32:1120-7.

39 Marrero JA, Fontana RJ, Barrat A, et al. Prognosis of hepatocellular carcinoma: comparison of 7 staging systems in an American cohort. Hepatology 2005;41:707-15.

40 Vitale A, Saracino E, Boccagni P, et al. Validation of the BCLC prognostic system in surgical hepatocellular cancer patients. Transplant Proc 2009;41:1260-3.

41 Nanashima A, Sumida Y, Abo T, et al. Modified Japan integrated staging is currently the best available staging system for hepatocellular carcinoma patients who have undergone hepatectomy. J Gastroenterol 2006;41:250-6.

42 Hsu C-Y, Hsia C-Y, Huang Y-H, et al. Selecting an optimal staging system for hepatocellular carcinoma: comparison of 5 currently used prognostic models. Cancer 2010;116:3006-14.

43 Huitzil-Melendez F-D, Capanu M, O'Reilly EM, et al. Advanced hepatocellular carcinoma: which staging systems best predict prognosis? J Clin Oncol 2010;28:2889-95.

44 Pugh RN, Murray-Lyon IM, Dawson JL, et al. Transection of the oesophagus for bleeding oesophageal varices. $\mathrm{Br} J$ Surg 1973;60:646-9.

45 Child CG, Turcotte JG. Surgery and portal hypertension. Major Probl Clin Surg 1964;1:1-85.

46 Llovet JM, Bustamante J, Castells A, et al. Natural history of untreated nonsurgical hepatocellular carcinoma: rationale for the design and evaluation of therapeutic trials. Hepatology 1999;29:62-7.

47 Abou-Alfa GK, Huitzil-Melendez F-D, O'Reilly EM, et al. Current management of advanced hepatocellular carcinoma. Gastrointest Cancer Res 2008:2:64-70.

48 Johnson PJ, Berhane S, Kagebayashi C, et al. Assessment of liver function in patients with hepatocellular carcinoma: a new evidence-based approach-the ALBI grade. J Clin Oncol 2015;33:550-8.

49 Gui B, Weiner AA, Nosher J, et al. Assessment of the albuminbilirubin (ALBI) grade as a prognostic indicator for hepatocellular carcinoma patients treated with radioembolization. Am J Clin Oncol 2018;41:861-6.

50 Pinato DJ, Sharma R, Allara E, et al. The ALBI grade provides objective hepatic reserve estimation across each BCLC stage of hepatocellular carcinoma. J Hepatol 2017;66:338-46.

51 Bruix J, Sherman M, Llovet JM, et al. Clinical management of hepatocellular carcinoma. conclusions of the Barcelona-2000 EASL conference. J Hepatol 2001;35:421-30.

52 Lederle FA, Pocha C. Screening for liver cancer: the rush to judgment. Ann Intern Med 2012;156:387-9.

53 Zhang J, Chen G, Zhang P, et al. The threshold of alpha-fetoprotein (AFP) for the diagnosis of hepatocellular carcinoma: a systematic review and meta-analysis. PLoS One 2020;15:e0228857.
54 Pardee AD, Shi J, Butterfield LH. Tumor-derived $\alpha$-fetoprotein impairs the differentiation and T cell stimulatory activity of human dendritic cells. J Immunol 2014;193:5723-32.

55 Capurro M, Wanless IR, Sherman M, et al. Glypican-3: a novel serum and histochemical marker for hepatocellular carcinoma. Gastroenterology 2003;125:89-97.

56 Shi D, Shi Y, Kaseb AO, et al. Chimeric antigen Receptor-Glypican-3 T-cell therapy for advanced hepatocellular carcinoma: results of phase I trials. Clin Cancer Res 2020;26:3979-89.

57 Best J, Bechmann LP, Sowa J-P, et al. GALAD score detects early hepatocellular carcinoma in an international cohort of patients with nonalcoholic steatohepatitis. Clin Gastroenterol Hepatol 2020;18:728-35.

58 Yang JD, Addissie BD, Mara KC, et al. GALAD score for hepatocellular carcinoma detection in comparison with liver ultrasound and proposal of GALADUS score. Cancer Epidemiol Biomarkers Prev 2019;28:531-8.

59 Kambhampati S, Bauer KE, Bracci PM, et al. Nivolumab in patients with advanced hepatocellular carcinoma and Child-Pugh class $\mathrm{B}$ cirrhosis: safety and clinical outcomes in a retrospective case series. Cancer 2019;125:3234-41.

60 Fessas P, Kaseb A, Wang Y, et al. Post-registration experience of nivolumab in advanced hepatocellular carcinoma: an international study. J Immunother Cancer 2020;8:e001033.

61 Kudo M, Matilla A, Santoro A, et al. CheckMate 040 cohort 5: a phase I/II study of nivolumab in patients with advanced hepatocellular carcinoma and Child-Pugh B cirrhosis. $J$ Hepatol 2021. doi:10.1016/j.jhep.2021.04.047. [Epub ahead of print: 12 May 2021].

62 FDA. FDA grants accelerated approval to nivolumab and ipilimumab combination for hepatocellular carcinoma. US: FDA, 2020.

63 El-Khoueiry AB, Yau T, Kang Y-K, et al. Nivolumab (NIVO) plus ipilimumab (IPI) combination therapy in patients (PTS) with advanced hepatocellular carcinoma (aHCC): long-term results from CheckMate 040. JCO 2021;39:269.

64 Yau T, Park JW, Finn RS, et al. CheckMate 459: a randomized, multi-center phase III study of nivolumab (NIVO) vs sorafenib (SOR) as first-line (1L) treatment in patients (PTS) with advanced hepatocellular carcinoma (aHCC). Ann Oncol 2019;30:v874-5.

65 Finn RS, Ryoo B-Y, Merle P, et al. Pembrolizumab as second-line therapy in patients with advanced hepatocellular carcinoma in KEYNOTE-240: a randomized, double-blind, phase III trial. J Clin Oncol 2020;38:193-202.

66 Finn RS, Qin S, Ikeda M, et al. IMbrave150: updated overall survival (OS) data from a global, randomized, open-label phase III study of atezolizumab (atezo) + bevacizumab (bev) versus sorafenib (SOR) in patients (PTS) with unresectable hepatocellular carcinoma (HCC). JCO 2021;39:267.

67 Enrico D, Paci A, Chaput N, et al. Antidrug antibodies against immune checkpoint blockers: impairment of drug efficacy or indication of immune activation? Clin Cancer Res 2020;26:787-92.

68 Peter R, Cheng A-L, Bernaards C. CT185 - Assessment of the impact of anti-drug antibodies on PK and clinical outcomes with atezolizumab + bevacizumab in HCC. AACR Annual Meeting, 2021.

69 Sangro B, Gomez-Martin C, de la Mata M, et al. A clinical trial of CTLA-4 blockade with tremelimumab in patients with hepatocellular carcinoma and chronic hepatitis C. J Hepatol 2013;59:81-8.

70 Qin S, Finn RS, Kudo M, et al. A phase 3, randomized, openlabel, multicenter study to compare the efficacy and safety of tislelizumab, an anti-PD-1 antibody, versus sorafenib as first-line treatment in patients with advanced hepatocellular carcinoma. JCO 2018:36:TPS3110. doi:10.1200/JCO.2018.36.15 suppl.TPS3110

71 Qin SK, Ren ZG, Meng ZQ, et al. A randomized multicentered phase II study to evaluate SHR-1210 (PD-1 antibody) in subjects with advanced hepatocellular carcinoma $(\mathrm{HCC})$ who failed or intolerable to prior systemic treatment. Ann Oncol 2018;29:mdy424. 029:viii719-20. doi:10.1093/annonc/mdy424.029

72 Wainberg ZA, Segal NH, Jaeger D, et al. Safety and clinical activity of durvalumab monotherapy in patients with hepatocellular carcinoma (HCC). JCO 2017;35:4071. doi:10.1200/ JCO.2017.35.15_suppl.4071

73 Squibb BM. Nivolumab highlights of prescribing information. US FDA: Drugs@FDA, 2020.

74 Kelley RK, Sangro B, Harris WP, et al. Efficacy, tolerability, and biologic activity of a novel regimen of tremelimumab $(T)$ in combination with durvalumab (D) for patients (PTS) with advanced hepatocellular carcinoma (aHCC). JCO 2020;38:4508. doi:10.1200/ JCO.2020.38.15 suppl.4508

75 Abou-Alfa GK, Chan SL, Furuse J, et al. A randomized, multicenter phase 3 study of durvalumab (D) and tremelimumab (T) as first-line treatment in patients with unresectable hepatocellular carcinoma 
(HCC): HIMALAYA study. JCO 2018;36:TPS4144-TPS doi:10.1200/ JCO.2018.36.15_suppl.TPS4144

76 Yau T, Zagonel V, Santoro A, et al. Nivolumab (NIVO) + ipilimumab (IPI) + cabozantinib (Cabo) combination therapy in patients (PTS) with advanced hepatocellular carcinoma (aHCC): results from CheckMate 040. JCO 2020;38:478.

77 Finn RS, Ikeda M, Zhu AX, et al. Phase lb study of lenvatinib plus pembrolizumab in patients with unresectable hepatocellular carcinoma. J Clin Oncol 2020;38:2960-70.

78 Llovet JM, Kudo M, Cheng A-L, et al. Lenvatinib (LEN) plus pembrolizumab (pembro) for the first-line treatment of patients (PTS) with advanced hepatocellular carcinoma (HCC): phase 3 LEAP-002 study. JCO 2019;37:TPS4152-TPS

79 Kudo M, Motomura K, Wada Y. First-Line avelumab+ axitinib in patients with advanced hepatocellular carcinoma: results from a phase 1B trial (VEGF liver 100). J Clin Oncol 2019;35.

80 Kelley RK, Cheng A-L, Braiteh FS, et al. Phase 3 (COSMIC-312) study of cabozantinib (C) in combination with atezolizumab (a) versus sorafenib (S) in patients (PTS) with advanced hepatocellular carcinoma (aHCC) who have not received previous systemic anticancer therapy. JCO 2019;37:TPS4157-TP

81 Greten TF, Mauda-Havakuk M, Heinrich B, et al. Combined locoregional-immunotherapy for liver cancer. J Hepatol 2019;70:999-1007.

82 Galluzzi L, Buqué A, Kepp O, et al. Immunological effects of conventional chemotherapy and targeted anticancer agents. Cancer Cell 2015;28:690-714.

83 Ayaru L, Pereira SP, Alisa A, et al. Unmasking of alpha-fetoproteinspecific CD4(+) T cell responses in hepatocellular carcinoma patients undergoing embolization. J Immunol 2007;178:1914-22.

84 Nobuoka D, Motomura Y, Shirakawa H, et al. Radiofrequency ablation for hepatocellular carcinoma induces glypican-3 peptidespecific cytotoxic T lymphocytes. Int J Oncol 2012;40:63-70.

85 Duffy AG, Ulahannan SV, Makorova-Rusher O, et al. Tremelimumab in combination with ablation in patients with advanced hepatocellular carcinoma. J Hepatol 2017;66:545-51.

86 Mazzaferro V, Llovet JM, Miceli R, et al. Predicting survival after liver transplantation in patients with hepatocellular carcinoma beyond the Milan criteria: a retrospective, exploratory analysis. Lancet Oncol 2009;10:35-43.

87 Munker S, De Toni EN. Use of checkpoint inhibitors in liver transplant recipients. United European Gastroenterol $J$ 2018;6:970-3.

88 Abdel-Wahab N, Safa H, Abudayyeh A, et al. Checkpoint inhibitor therapy for cancer in solid organ transplantation recipients: an institutional experience and a systematic review of the literature. $J$ Immunother Cancer 2019;7:106.

89 Greten TF, Forner A, Korangy F, et al. A phase II open label trial evaluating safety and efficacy of a telomerase peptide vaccination in patients with advanced hepatocellular carcinoma. BMC Cancer 2010;10:209.

90 Sawada Y, Yoshikawa T, Nobuoka D, et al. Phase I trial of a glypican-3-derived peptide vaccine for advanced hepatocellular carcinoma: immunologic evidence and potential for improving overall survival. Clin Cancer Res 2012;18:3686-96.

91 Sawada Y, Yoshikawa T, Ofuji K, et al. Phase II study of the GPC3derived peptide vaccine as an adjuvant therapy for hepatocellular carcinoma patients. Oncoimmunology 2016;5:e1129483-e.

92 Butterfield LH, Meng WS, Koh A, et al. T cell responses to HLA$A^{*} 0201$-restricted peptides derived from human alpha fetoprotein. $J$ Immunol 2001;166:5300-8.

93 Butterfield LH, Ribas A, Meng WS, et al. T-cell responses to HLA-A*0201 immunodominant peptides derived from alphafetoprotein in patients with hepatocellular cancer. Clin Cancer Res 2003;9:5902-8

94 Butterfield LH, Ribas A, Dissette VB, et al. A phase I/II trial testing immunization of hepatocellular carcinoma patients with dendritic cells pulsed with four alpha-fetoprotein peptides. Clin Cancer Res 2006;12:2817-25.

95 Peng B-G, Liang L-J, He Q, et al. Tumor vaccine against recurrence of hepatocellular carcinoma. World J Gastroenterol 2005;11:700-4.

96 Palmer DH, Midgley RS, Mirza N, et al. A phase II study of adoptive immunotherapy using dendritic cells pulsed with tumor lysate in patients with hepatocellular carcinoma. Hepatology 2009:49:124-32.

97 Bourinbaiar AS, Chinburen J, Batchuluun P, et al. Interim results from ongoing phase III placebo-controlled, randomized trial of hepcortespenlisimut-L for advanced hepatocellular carcinoma indication. Hepatoma Res 2020;2020:2.

98 Pardi N, Hogan MJ, Porter FW, et al. mRNA vaccines - a new era in vaccinology. Nat Rev Drug Discov 2018;17:261-79.
99 Miao L, Zhang Y, Huang L. mRNA vaccine for cancer immunotherapy. Mol Cancer 2021;20:41.

100 Buonaguro L, Mayer-Mokler A, Accolla R, et al. HepaVac-101 first-in-man therapeutic cancer vaccine phase I/II clinical trial for hepatocellular carcinoma patients. JCO 2018;36:TPS3135-TPS

101 Sullivan KM, Dykewicz CA, Longworth DL, et al. Preventing opportunistic infections after hematopoietic stem cell transplantation: the centers for disease control and prevention, infectious diseases Society of America, and American Society for blood and marrow transplantation practice guidelines and beyond. Hematology Am Soc Hematol Educ Program 2001:392-421.

102 Schmidt-Wolf IG, Negrin RS, Kiem HP, et al. Use of a SCID mouse/ human lymphoma model to evaluate cytokine-induced killer cells with potent antitumor cell activity. J Exp Med 1991:174:139-49.

103 Schmidt-Wolf IG, Lefterova P, Mehta BA, et al. Phenotypic characterization and identification of effector cells involved in tumor cell recognition of cytokine-induced killer cells. Exp Hematol 1993;21:1673-9.

104 Lee JH, Lee J-H, Lim Y-S, et al. Adjuvant immunotherapy with autologous cytokine-induced killer cells for hepatocellular carcinoma. Gastroenterology 2015;148:1383-91.

105 Ma Y, Xu Y-C, Tang L, et al. Cytokine-induced killer (CIK) cell therapy for patients with hepatocellular carcinoma: efficacy and safety. Exp Hematol Oncol 2012;1:11.

106 Alnaggar M, Lin M, Mesmar A, et al. Allogenic natural killer cell immunotherapy combined with irreversible electroporation for stage IV hepatocellular carcinoma: survival outcome. Cell Physiol Biochem 2018;48:1882-93.

107 Lin M, Liang S, Wang X, et al. Cryoablation combined with allogenic natural killer cell immunotherapy improves the curative effect in patients with advanced hepatocellular cancer. Oncotarget 2017:8:81967-77.

108 Couto OFM, Dvorchik I, Carr BI. Causes of death in patients with unresectable hepatocellular carcinoma. Dig Dis Sci 2007;52:3285-9.

109 Bersanelli M, Brighenti M, Buti S, et al. Patient performance status and cancer immunotherapy efficacy: a meta-analysis. Med Oncol 2018;35:132

110 Butaney M, Satkunasivam R, Goldberg H, et al. Analysis of heterogeneity in survival benefit of immunotherapy in oncology according to patient demographics and performance status: a systematic review and meta-analysis of overall survival data. Am J Clin Oncol 2020;43:193-202.

111 Kudo M. Recent advances in systemic therapy for hepatocellular carcinoma in an aging Society: 2020 update. Liver Cancer 2020;9:640-62.

112 Hershman DL, Wright JD, Lim E, et al. Contraindicated use of bevacizumab and toxicity in elderly patients with cancer. $J$ Clin Oncol 2013:31:3592-9.

113 Dika IE, Harding JJ, Abou-Alfa GK. Hepatocellular carcinoma in patients with HIV. Curr Opin HIV AIDS 2017;12:20-5.

$114 \mathrm{Hu}$ J, Liu K, Luo J. HIV-HBV and HIV-HCV coinfection and liver cancer development. Cancer Treat Res 2019;177:231-50.

115 Pinato DJ, Allara E, Chen T-Y, et al. Influence of HIV infection on the natural history of hepatocellular carcinoma: results from a global Multicohort study. J Clin Oncol 2019;37:296-304.

116 Uldrick TS, Gonçalves PH, Abdul-Hay M, et al. Assessment of the safety of pembrolizumab in patients with HIV and advanced cancer-a phase 1 study. JAMA Oncol 2019:5:1332-9.

117 González-Cao M, Moran T, Dalmau J. Phase II study of durvalumab (MEDI4736) in cancer patients HIV-1-infected. J Clin Oncol 2019;39. doi:10.1200/JCO.2019.37.15 suppl.2501

118 Boland P, Pavlick AC, Weber J, et al. Immunotherapy to treat malignancy in patients with pre-existing autoimmunity. $J$ Immunother Cancer 2020;8:e000356.

119 Abdel-Wahab N, Shah M, Lopez-Olivo MA, et al. Use of immune checkpoint inhibitors in the treatment of patients with cancer and preexisting autoimmune disease: a systematic review. Ann Intern Med 2018;168:121-30

120 Arbour KC, Mezquita L, Long N, et al. Impact of baseline steroids on efficacy of programmed cell death-1 and programmed DeathLigand 1 blockade in patients with non-small-cell lung cancer. $J$ Clin Oncol 2018;36:2872-8.

121 Petrelli F, Signorelli D, Ghidini M, et al. Association of steroids use with survival in patients treated with immune checkpoint inhibitors: a systematic review and meta-analysis. Cancers 2020;12. doi:10.3390/cancers12030546. [Epub ahead of print: 27 Jan 2020].

122 Rich NE, Hester C, Odewole M, et al. Racial and ethnic differences in presentation and outcomes of hepatocellular carcinoma. Clin Gastroenterol Hepatol 2019;17:551-9. 
123 Bartlett C, Doyal L, Ebrahim S. The causes and effects of sociodemographic exclusions from clinical trials. Health Technol Assess 2005;9:1-152.

124 Oh SS, Galanter J, Thakur N, et al. Diversity in clinical and biomedical research: a promise yet to be fulfilled. PLoS Med 2015;12:e1001918.

125 Kaseb AO, Tran Cao HS, Mohamed YI, et al. Final results of a randomized, open label, perioperative phase II study evaluating nivolumab alone or nivolumab plus ipilimumab in patients with resectable HCC. JCO 2020;38:4599.

126 Pinato DJ, Cortellini A, Sukumaran A, et al. PRIME-HCC: phase Ib study of neoadjuvant ipilimumab and nivolumab prior to liver resection for hepatocellular carcinoma. BMC Cancer 2021;21:301.

127 Yarchoan M, Zhu Q, Durham JN, et al. Feasibility and efficacy of neoadjuvant cabozantinib and nivolumab in patients with borderline resectable or locally advanced hepatocellular carcinoma (HCC). JCO 2021;39:335.

128 Xie Y. Hepatitis B virus-associated hepatocellular carcinoma. Adv Exp Med Biol 2017;1018:11-21.

129 Harrod E, Moctezuma-Velazquez C, Gurakar A, et al. Management of concomitant hepatocellular carcinoma and chronic hepatitis C: a review. Hepatoma Res 2019;2019:28.

130 Shah NJ, Al-Shbool G, Blackburn M, et al. Safety and efficacy of immune checkpoint inhibitors (ICls) in cancer patients with HIV, hepatitis B, or hepatitis C viral infection. $J$ Immunother Cancer 2019;7:353

131 Li B, Yan C, Zhu J, et al. Anti-Pd-1/Pd-L1 blockade immunotherapy employed in treating hepatitis $B$ virus infection-related advanced hepatocellular carcinoma: a literature review. Front Immunol 2020;11:1037.

132 Pfister D, Núñez NG, Pinyol R, et al. NASH limits antitumour surveillance in immunotherapy-treated HCC. Nature 2021:592:450-6.

133 Ho WJ, Danilova L, Lim SJ, et al. Viral status, immune microenvironment and immunological response to checkpoint inhibitors in hepatocellular carcinoma. $J$ Immunother Cancer 2020;8.

134 Richard Finn S S, Masafumilkeda PRG, Ducreux M. IMbrave150: updated efficacy and safety by risk status in patients (PTS) receiving Atezolizumab (atezo) + bevacizumab (bev) vs sorafenib (SOR) as first-line treatment for unresectable hepatocellular carcinoma (HCC). AACR Annual Meeting, 2021.

135 FDA. TECENTRIQ highlights of prescribing information. Drugs@FDA

136 Zhu AX, Kang Y-K, Yen C-J, et al. Ramucirumab after sorafenib in patients with advanced hepatocellular carcinoma and increased $\alpha$-fetoprotein concentrations (REACH-2): a randomised, double-blind, placebo-controlled, phase 3 trial. Lancet Oncol 2019;20:282-96.

137 Sangro B, Park J, Finn R, et al. LBA-3 CheckMate 459: longterm (minimum follow-up 33.6 months) survival outcomes with nivolumab versus sorafenib as first-line treatment in patients with advanced hepatocellular carcinoma. Ann of Oncol 2020;31:S241-2.

138 Sánchez-Paulete AR, Cueto FJ, Martínez-López M, et al. Cancer immunotherapy with immunomodulatory Anti-CD137 and anti-PD-1 monoclonal antibodies requires BATF3-Dependent dendritic cells. Cancer Discov 2016;6:71-9.

139 Ruiz de Galarreta M, Bresnahan E, Molina-Sánchez P, et al. $\beta$-Catenin Activation Promotes Immune Escape and Resistance to Anti-PD-1 Therapy in Hepatocellular Carcinoma. Cancer Discov 2019;9:1124-41.

140 Harding JJ, Nandakumar S, Armenia J, et al. Prospective genotyping of hepatocellular carcinoma: clinical implications of next-generation sequencing for matching patients to targeted and immune therapies. Clin Cancer Res 2019;25:2116-26.

141 Feun LG, Li Y-Y, Wu C, Wangpaichitr M, et al. Phase 2 study of pembrolizumab and circulating biomarkers to predict anticance response in advanced, unresectable hepatocellular carcinoma. Cancer 2019;125:3603-14.

142 Sangro B, Melero I, Wadhawan S, et al. Association of inflammatory biomarkers with clinical outcomes in nivolumab-treated patients with advanced hepatocellular carcinoma. $J$ Hepatol 2020;73:1460-9.

143 Wang Z, Xu Y, Gong F, et al. 997P PD-L1 protein expression as a predictor of response to immune checkpoint inhibitor (ICI) in hepatocellular carcinoma (HCC): a meta-analysis. Ann Oncol 2020;31:S694.

144 Pinato DJ, Mauri FA, Spina P, et al. Clinical implications of heterogeneity in PD-L1 immunohistochemical detection in hepatocellular carcinoma: the Blueprint-HCC study. Br J Cancer 2019:120:1033-6.
145 Sia D, Jiao Y, Martinez-Quetglas I, et al. Identification of an Immune-specific class of hepatocellular carcinoma, based on molecular features. Gastroenterology 2017;153:812-26.

146 Meyer T, Melero I, Yau T, et al. Hepatic safety and biomarker assessments in sorafenib-experienced patients with advanced hepatocellular carcinoma treated with nivolumab in the CheckMate-040 study. J Hepatol 2018;68:S16.

147 Le DT, Uram JN, Wang H, et al. Pd-1 blockade in tumors with mismatch-repair deficiency. N Engl J Med 2015;372:2509-20.

148 Le DT, Kim TW, Van Cutsem E, et al. Phase II open-label study of pembrolizumab in treatment-refractory, microsatellite InstabilityHigh/Mismatch repair-deficient metastatic colorectal cancer: KEYNOTE-164. J Clin Oncol 2020;38:11-19.

149 Nanda R, Chow LQM, Dees EC, et al. Pembrolizumab in patients with advanced triple-negative breast cancer: phase $\mathrm{lb}$ KEYNOTE-012 study. J Clin Oncol 2016;34:2460-7.

150 Rugo HS, Delord J-P, Im S-A. Safety and antitumor activity of pembrolizumab in patients with estrogen receptor-positive/human epidermal growth factor receptor 2-negative advanced breast cancer. Clin Cancer Res.

151 Marabelle A, Le DT, Ascierto PA, et al. Efficacy of pembrolizumab in patients with Noncolorectal high microsatellite Instability/Mismatch repair-deficient cancer: results from the phase II KEYNOTE-158 study. J Clin Oncol 2020;38:1-10.

152 US FDA. FDA approves pembrolizumab for adults and children with TMB-H solid tumors [press release]. Drug Approvals and Databases, 2020

$153 \mathrm{Kim}$ JY, Kronbichler A, Eisenhut M, et al. Tumor mutational burden and efficacy of immune checkpoint inhibitors: a systematic review and meta-analysis. Cancers 2019;11:1798.

154 Osipov A, Lim SJ, Popovic A, et al. Tumor Mutational Burden, Toxicity, and Response of Immune Checkpoint Inhibitors Targeting PD(L)1, CTLA-4, and Combination: A Meta-regression Analysis. Clin Cancer Res 2020;26:4842-51.

155 Ang C, Klempner SJ, Ali SM, et al. Prevalence of established and emerging biomarkers of immune checkpoint inhibitor response in advanced hepatocellular carcinoma. Oncotarget 2019;10:4018-25.

156 Chiappini F, Gross-Goupil M, Saffroy R, et al. Microsatellite instability mutator phenotype in hepatocellular carcinoma in nonalcoholic and non-virally infected normal livers. Carcinogenesis 2004;25:541-7.

157 Zheng Y-B, Zhao W, Liu B, et al. The blood neutrophil-tolymphocyte ratio predicts survival in patients with advanced hepatocellular carcinoma receiving sorafenib. Asian Pac J Cancer Prev 2013;14:5527-31.

158 Hong YM, Yoon KT, Hwang TH, et al. Changes in the neutrophil-tolymphocyte ratio predict the prognosis of patients with advanced hepatocellular carcinoma treated with sorafenib. Eur J Gastroenterol Hepatol 2019;31:1250-5

159 Dharmapuri S, Özbek U, Lin J-Y, et al. Predictive value of neutrophil to lymphocyte ratio and platelet to lymphocyte ratio in advanced hepatocellular carcinoma patients treated with anti-PD-1 therapy. Cancer Med 2020;9:4962-70.

160 Dapito DH, Mencin A, Gwak G-Y, et al. Promotion of hepatocellular carcinoma by the intestinal microbiota and TLR4. Cancer Cell 2012;21:504-16.

161 Yoshimoto S, Loo TM, Atarashi K, et al. Obesity-induced gut microbial metabolite promotes liver cancer through senescence secretome. Nature 2013;499:97-101.

162 Routy B, Le Chatelier E, Derosa L, et al. Gut microbiome influences efficacy of PD-1-based immunotherapy against epithelial tumors. Science 2018;359:91-7.

163 Derosa L, Hellmann MD, Spaziano M, et al. Negative association of antibiotics on clinical activity of immune checkpoint inhibitors in patients with advanced renal cell and non-small-cell lung cancer. Ann Oncol 2018;29:1437-44.

164 Pinato DJ, Howlett S, Ottaviani D, et al. Association of prior antibiotic treatment with survival and response to immune checkpoint inhibitor therapy in patients with cancer. JAMA Oncol 2019;5:1774-8

165 Zheng Y, Wang T, Tu X, et al. Gut microbiome affects the response to anti-PD-1 immunotherapy in patients with hepatocellular carcinoma. J Immunother Cancer 2019;7:193.

166 Daly LE, Power DG, O'Reilly Áine, et al. The impact of body composition parameters on ipilimumab toxicity and survival in patients with metastatic melanoma. Br J Cancer 2017;116:310-7.

167 Rollins KE, Tewari N, Ackner A, et al. The impact of sarcopenia and myosteatosis on outcomes of unresectable pancreatic cancer or distal cholangiocarcinoma. Clin Nutr 2016;35:1103-9. 
168 Wang S, Cowley LA, Liu X-S. Sex differences in cancer immunotherapy efficacy, biomarkers, and therapeutic strategy. Molecules 2019;24:3214

169 Valpione S, Pasquali S, Campana LG, et al. Sex and interleukin-6 are prognostic factors for autoimmune toxicity following treatment with anti-CTLA4 blockade. J Trans/ Med 2018;16:94.

170 Dubin K, Callahan MK, Ren B, et al. Intestinal microbiome analyses identify melanoma patients at risk for checkpoint-blockade-induced colitis. Nat Commun 2016:7:10391.

171 Tarhini AA, Zahoor H, Lin Y, et al. Baseline circulating IL-17 predicts toxicity while TGF- $\beta 1$ and IL-10 are prognostic of relapse in ipilimumab neoadjuvant therapy of melanoma. $J$ Immunother Cancer 2015;3:39.

172 de Moel EC, Rozeman EA, Kapiteijn EH, et al. Autoantibody development under treatment with Immune-Checkpoint inhibitors. Cancer Immunol Res 2019;7:6-11.

173 Yoest JM. Clinical features, predictive correlates, and pathophysiology of immune-related adverse events in immune checkpoint inhibitor treatments in cancer: a short review. Immunotargets Ther 2017;6:73-82.

174 Wang DY, Salem J-E, Cohen JV, et al. Fatal toxic effects associated with immune checkpoint inhibitors: a systematic review and metaanalysis. JAMA Oncol 2018;4:1721-8.

175 Xing P, Zhang F, Wang G, et al. Incidence rates of immune-related adverse events and their correlation with response in advanced solid tumours treated with NIVO or NIVO+IPI: a systematic review and meta-analysis. J Immunother Cancer 2019;7:341.

176 David Pinato J J, Marron TU, et al. Treatment-related toxicity predicts for improved outcome in patients with hepatocellular carcinoma (HCC) treated with immune checkpoint inhibitor therapy. $J$ Hepatol 2020;73:S19-57.

177 Brahmer JR, Abu-Sbeih H, Ascierto PA, et al. Society for immunotherapy of cancer (SITC) clinical practice guideline on immune checkpoint inhibitor-related adverse events. J Immunother Cancer 2021;9:e002435.

178 Yau T, Kang Y-K, Kim T-Y, et al. Efficacy and safety of nivolumab plus ipilimumab in patients with advanced hepatocellular carcinoma previously treated with sorafenib: the CheckMate 040 randomized clinical trial. JAMA Oncol 2020;6::e204564e:e204564.

179 Sangro B, Chan SL, Meyer T, et al. Diagnosis and management of toxicities of immune checkpoint inhibitors in hepatocellular carcinoma. J Hepatol 2020;72:320-41.

180 Organization WH. WHO Handbook for reporting results of cancer treatment. World Health Organization, 1979.

181 Therasse P, Arbuck SG, Eisenhauer EA, et al. New guidelines to evaluate the response to treatment in solid tumors. J Natl Cancer Inst 2000;92:205-16.

182 Gonzalez-Guindalini FD, Botelho MPF, Harmath CB, et al. Assessment of liver tumor response to therapy: role of quantitative imaging. Radiographics 2013;33:1781-800.

183 Llovet JM, Di Bisceglie AM, Bruix J, et al. Design and endpoints of clinical trials in hepatocellular carcinoma. $J$ Natl Cancer Inst 2008;100:698-711.

184 Lencioni R, Llovet JM. Modified RECIST (mRECIST) assessment for hepatocellular carcinoma. Semin Liver Dis 2010;30:52-60.

185 Vincenzi B, Di Maio M, Silletta M, et al. Prognostic relevance of objective response according to EASL criteria and mRECIST criteria in hepatocellular carcinoma patients treated with locoregional therapies: a Literature-Based meta-analysis. PLoS One 2015;10:e0133488-e.

186 Prajapati HJ, Spivey JR, Hanish SI, et al. mRECIST and EASL responses at early time point by contrast-enhanced dynamic MRI predict survival in patients with unresectable hepatocellular carcinoma (HCC) treated by doxorubicin drug-eluting beads transarterial chemoembolization (DEB TACE). Ann Oncol 2013;24:965-73.

187 Kudo M, Finn RS, Qin S, et al. Analysis of survival and objective response (or) in patients with hepatocellular carcinoma in a phase III study of lenvatinib (reflect). JCO 2019;37:186.

188 Lencioni R, Montal R, Torres F, et al. Objective response by mRECIST as a predictor and potential surrogate end-point of overall survival in advanced HCC. $J$ Hepatol 2017;66:1166-72.

189 Grierson P, Crites D, Ruzinova MB, et al. Distinct clinical and magnetic resonance features of metastatic hepatocellular carcinoma treated with pembrolizumab: a case report of late response after pseudoprogression. Hepatol Commun 2018;2:148-51.

190 Dromain C, Beigelman C, Pozzessere C, et al. Imaging of tumour response to immunotherapy. Eur Radiol Exp 2020;4:2.
191 Borcoman E, Nandikolla A, Long G, et al. Patterns of response and progression to immunotherapy. Am Soc Clin Oncol Educ Book 2018;38:169-78.

192 Frelaut M, Le Tourneau C, Borcoman E. Hyperprogression under immunotherapy. Int J Mol Sci 2019;20:2674.

193 Wong DJ, Lee J, Choo SP, et al. Hyperprogressive disease in hepatocellular carcinoma with immune checkpoint inhibitor use: a case series. Immunotherapy 2019;11:167-75.

194 Chan SL. Hyperprogression in hepatocellular carcinoma: illusion or reality? J Hepatol 2021;74:269-71.

195 Yeo W, Mo FKF, Koh J, et al. Quality of life is predictive of survival in patients with unresectable hepatocellular carcinoma. Ann Oncol 2006;17:1083-9.

196 Bonnetain F, Paoletti X, Collette S, et al. Quality of life as a prognostic factor of overall survival in patients with advanced hepatocellular carcinoma: results from two French clinical trials. Qual Life Res 2008;17:831-43.

197 Diouf M, Filleron T, Barbare J-C, et al. The added value of quality of life (QOL) for prognosis of overall survival in patients with palliative hepatocellular carcinoma. J Hepatol 2013;58:509-21.

198 Bultz BD, Carlson LE. Emotional distress: the sixth vital sign-future directions in cancer care. Psychooncology 2006;15:93-5.

199 Zabora J, BrintzenhofeSzoc K, Curbow B, et al. The prevalence of psychological distress by cancer site. Psychooncology 2001;10:19-28.

200 Vaughn-Sandler V, Sherman C, Aronsohn A, et al. Consequences of perceived stigma among patients with cirrhosis. Dig Dis Sci 2014;59:681-6.

201 Squibb B-M. U.S. survey of liver cancer caregivers. 24 Jul 2017 - 31 Aug 2017, 2017.

202 Philbin MM, Erby LAH, Lee S, et al. Hepatitis B and liver cancer among three Asian American sub-groups: a focus group inquiry. $J$ Immigr Minor Health 2012;14:858-68.

203 Li D, Tang T, Patterson M, et al. The impact of hepatitis B knowledge and stigma on screening in Canadian Chinese persons. Can J Gastroenterol 2012;26:705094

204 Kumar M, Panda D. Role of supportive care for terminal stage hepatocellular carcinoma. J Clin Exp Hepatol 2014;4:S130-9.

205 Woodrell CD, Hansen L, Schiano TD, et al. Palliative care for people with hepatocellular carcinoma, and specific benefits for older adults. Clin Ther 2018;40:512-25.

206 Wong A, Billett A, Milne D. Balancing the hype with reality: what do patients with advanced melanoma consider when making the decision to have immunotherapy? Oncologist 2019;24:e1190-6.

207 Temel JS, Greer JA, Muzikansky A, et al. Early palliative care for patients with metastatic non-small-cell lung cancer. N Engl J Med Overseas Ed 2010;363:733-42.

208 Ufere NN, Donlan J, Waldman L, et al. Barriers to use of palliative care and advance care planning discussions for patients with endstage liver disease. Clin Gastroenterol Hepatol 2019;17:2592-9.

209 Walling AM, Wenger NS. Palliative care and end-stage liver disease. Clin Gastroenterol Hepatol 2014;12:699-700.

210 Poonja Z, Brisebois A, van Zanten SV, et al. Patients with cirrhosis and denied liver transplants rarely receive adequate palliative care or appropriate management. Clin Gastroenterol Hepatol 2014;12:692-8.

211 Nishijima TF, Shachar SS, Nyrop KA, et al. Safety and tolerability of PD-1/PD-L1 inhibitors compared with chemotherapy in patients with advanced cancer: a meta-analysis. Oncologist 2017;22:470-9.

212 Meilleur A, Subramanian SV, Plascak JJ, et al. Rural residence and cancer outcomes in the United States: issues and challenges. Cancer Epidemiol Biomarkers Prev 2013;22:1657-67.

213 Ward MM, Ullrich F, Matthews K, et al. Access to chemotherapy services by availability of local and visiting oncologists. $J$ Oncol Pract 2014;10:26-31

214 Liu G, Franssen E, Fitch MI, et al. Patient preferences for oral versus intravenous palliative chemotherapy. J Clin Oncol 1997;15:110-5.

215 Blazeby JM, Currie E, Zee BCY, et al. Development of a questionnaire module to supplement the EORTC QLQ-C30 to assess quality of life in patients with hepatocellular carcinoma, the EORTC QLQ-HCC18. Eur J Cancer 2004;40:2439-44.

216 Heffernan N, Cella D, Webster K, et al. Measuring health-related quality of life in patients with hepatobiliary cancers: the functional assessment of cancer therapy-hepatobiliary questionnaire. J Clin Oncol 2002;20:2229-39.

217 Yount S, Cella D, Webster K, et al. Assessment of patient-reported clinical outcome in pancreatic and other hepatobiliary cancers: the fact hepatobiliary symptom index. J Pain Symptom Manage 2002;24:32-44. 
218 Chonghua W, Jiqian F, Canzhen Z. Development and evaluation of a quality of life scale for patients of liver cancer. Chin J Behav Med Sci 1998;7:170-3.

219 Fan S-Y, Eiser C, Ho M-C. Health-Related quality of life in patients with hepatocellular carcinoma: a systematic review. Clin Gastroenterol Hepatol 2010;8:559-64.

220 Gandhi S, Khubchandani S, lyer R. Quality of life and hepatocellular carcinoma. J Gastrointest Oncol 2014;5:296-317.

221 Fan S-Y, Eiser C. Illness experience in patients with hepatocellular carcinoma: an interpretative phenomenological analysis study. Eur J Gastroenterol Hepatol 2012;24:203-8.

222 Fan S-Y, Eiser C, Ho M-C, et al. Health-related quality of life in patients with hepatocellular carcinoma: the mediation effects of illness perceptions and coping. Psychooncology 2013;22:1353-60.

223 Christian-Miller N, Frenette C. Hepatocellular cancer pain: impact and management challenges. J Hepatocell Carcinoma 2018;5:75-80.

224 Trevisani F, Frigerio M, Santi V, et al. Hepatocellular carcinoma in non-cirrhotic liver: a reappraisal. Dig Liver Dis 2010;42:341-7.

225 Imani F, Motavaf M, Safari S, et al. The therapeutic use of analgesics in patients with liver cirrhosis: a literature review and evidence-based recommendations. Hepat Mon 2014;14:e23539-e.

226 Rakoski M, Goyal P, Spencer-Safier M, et al. Pain management in patients with cirrhosis. Clin Liver Dis 2018;11:135-40.

227 Tran G, Zafar SY. Financial toxicity and implications for cancer care in the era of molecular and immune therapies. Ann Trans/ Med 2018;6:166.

228 Glode AE, May MB. Rising cost of cancer pharmaceuticals: cost issues and interventions to control costs. Pharmacotherapy 2017;37:85-93.
229 Mailankody S, Prasad V. Five years of cancer drug approvals: innovation, efficacy, and costs. JAMA Oncol 2015;1:539-40.

230 Zafar SY, Peppercorn JM, Schrag D, et al. The financial toxicity of cancer treatment: a pilot study assessing out-of-pocket expenses and the insured cancer patient's experience. Oncologist 2013;18:381-90.

231 Yabroff KR, Dowling EC, Guy GP, et al. Financial hardship associated with cancer in the United States: findings from a population-based sample of adult cancer survivors. J Clin Oncol 2016;34:259-67.

232 Fenn KM, Evans SB, McCorkle R, et al. Impact of financial burden of cancer on survivors' quality of life. $J$ Oncol Pract 2014;10:332-8.

233 Thein $\mathrm{H}-\mathrm{H}$, Isaranuwatchai W, Campitelli MA, et al. Health care costs associated with hepatocellular carcinoma: a populationbased study. Hepatology 2013;58:1375-84.

234 Galle PR, Finn RS, Qin S, et al. Patient-Reported outcomes (pros) from the phase III IMbrave150 trial of atezolizumab (atezo) + bevacizumab (bev) vs sorafenib (SOR) as first-line treatment (tx) for patients (PTS) with unresectable hepatocellular carcinoma (HCC). JCO 2020;38:476.

235 Ryoo B-Y, Merle P, Kulkarni AS, et al. Health-related qualityof-life impact of pembrolizumab versus best supportive care in previously systemically treated patients with advanced hepatocellular carcinoma: KEYNOTE-240. Cancer 2021;127:865-74.

236 (Liver) ACoRCoL-R. Liver reporting \& data system (LI-RADS): American College of Radiology. Liver reporting \& data system (LIRADS) 